\title{
Proteomic analysis of apricot fruit during ripening
}

\author{
Chiara D’Ambrosio ${ }^{a, 1}$, Simona Arena ${ }^{a, 1}$, Mariapina Rocco ${ }^{b, 1}$, Francesca Verrillo ${ }^{b}$, \\ Gianfranco Novi ${ }^{a}$, Vincenzo Viscosic ${ }^{c}$ Mauro Marra ${ }^{d, *}$, Andrea Scaloni ${ }^{a, * *}$ \\ aproteomics \& Mass Spectrometry Laboratory, ISPAAM, National Research Council, Naples 80147, Italy \\ ${ }^{\mathrm{b}}$ Department of Biological, Geological and Environmental Sciences, University of Sannio, Benevento 82100, Italy \\ ${ }^{\circ}$ Comprehensive Institute G. Pepe, Castelmauro (Campobasso) 86031, Italy \\ dDepartment of Biology, University of Rome "Tor Vergata", Rome 00100, Italy
}

\section{A R T I C L E I N F O}

Article history:

Received 22 June 2012

Accepted 5 November 2012

Available online 23 November 2012

Keywords:

Apricot

Fruit ripening

Proteomics

Prunus armeniaca

\begin{abstract}
A B S T R A C T
Ripening of climacteric fruits involves a complex network of biochemical and metabolic changes that make them palatable and rich in nutritional and health-beneficial compounds. Since fruit maturation has a profound impact on human nutrition, it has been recently the object of increasing research activity by holistic approaches, especially on model species. Here we report on the original proteomic characterization of ripening in apricot, a widely cultivated species of temperate zones appreciated for its taste and aromas, whose cultivation is yet hampered by specific limitations. Fruits of Prunus armeniaca cv. Vesuviana were harvested at three ripening stages and proteins extracted and resolved by $1 \mathrm{D}$ and $2 \mathrm{D}$ electrophoresis. Whole lanes from 1D gels were subjected to shot-gun analysis that identified 245 gene products, showing preliminary qualitative differences between maturation stages. In parallel, differential analysis of 2D proteomic maps highlighted 106 spots as differentially represented among variably ripen fruits. Most of these were further identified by means of MALDI-TOF-PMF and nanoLC-ESI-LIT-MS/MS as enzymes involved in main biochemical processes influencing metabolic/structural changes occurring during maturation, i.e. organic acids, carbohydrates and energy metabolism, ethylene biosynthesis, cell wall restructuring and stress response, or as protein species linkable to peculiar fruit organoleptic characteristics. In addition to originally present preliminary information on the main biochemical changes that characterize apricot ripening, this study also provides indications for future marker-assisted selection breeding programs aimed to ameliorate fruit quality.
\end{abstract}

(C) 2012 Published by Elsevier B.V.

\section{Introduction}

Common apricot (Prunus armeniaca L.) is a member of the Rosaceae family; most of the apricot varieties cultivated for fruit production belong to this species, which originated in Central Asia [1] and was then disseminated in Middle East, Mediterranean basin and Northern Europe areas. Adaptation to different environments resulted in significant phenotypic diversification, so that at least four major ecogeographical groups are recognized [2]. Production of apricot for different uses, as fresh or processed fruits, is

\footnotetext{
* Correspondence to: M. Marra, Department of Biology, University of Rome "Tor Vergata", via della Ricerca Scientifica 1, 00133 Rome, Italy.

** Correspondence to: A. Scaloni, Proteomics \& Mass Spectrometry Laboratory, ISPAAM, National Research Council, via Argine 1085, 80147 Naples, Italy.

E-mail addresses: Mauro.Marra@uniroma2.it (M. Marra), andrea.scaloni@ispaam.cnr.it (A. Scaloni).

${ }^{1}$ These authors gave an equal contribution to this work.
} 
economically very relevant (3,210,194 MT in 2009, according to FAO data) in the Mediterranean countries and in Italy, where almost 60 and $15 \%$ of the world harvest is generated, respectively. Apricot cultivation is hampered by some specific problems, such as high sensitivity to diseases (brown rot and sharka) or environmental stresses (spring frost), insufficient fruit quality or ripeness, as well as restricted harvest period. Consequently, breeding programs have been undertaken in different countries to overcome these limitations. In this respect, local cultivars are a source of genetic diversity, exploitable to select new varieties with improved agronomical traits. Whereas cultivars of the European ecogeographical group have a restricted genetic base [3], the Italian germplasm retains a high level of genetic diversity [2].

Breeding of fruit trees is exceedingly time-consuming and the availability of biomarkers linked to agronomical favorable traits can greatly improve process efficiency. As far as apricot, up to now molecular markers have been identified by isozyme analysis [3], amplified fragment length polymorphism [4] or microsatellites [5] and they have been used to investigate genetic diversity. Extensive expressed sequence tags (ESTs) collections or microarrays have greatly extended genetic information on vegetables or fruit-producing species, including peach and apricot. In this context, Grimplet and coworkers generated a collection of 13,006 ESTs from P. armeniaca mesocarp at different stages of ripening [6], while a bioinformatic database of integrated genetic information for Rosaceae (http://www.rosaceae.org) is available since 2008, which contains data on peach, apple and strawberry genomes. However, functional genomic studies have demonstrated that information deriving from nucleotide data do not necessarily match the corresponding translated protein complement at a precise organism physiological moment (e.g. fruit development or ripening) [7]; in fact, different modifications may affect gene products, including post-transcriptional, co-translational and degradative ones, along with environmental factors. Integration of genomic with proteomic data is hence highly desirable, in order to better clarify underlying molecular mechanisms, as well as to identify reliable molecular markers for crop breeding or amelioration.

Fruit ripening is a complex physiological process with a remarkable impact on human nutrition; occurring biochemical changes eventually make fleshy fruits palatable and rich in nutritional and health-beneficial compounds. Biochemical processes include the degradation of chlorophyll and starch, the biosynthesis of pigments and volatile compounds, the accumulation of sugars and organic acids, as well as cell wall softening $[8,9]$. Detailed comprehension of genetic regulatory elements is central for a full understanding of fruit ripening; in this context, proteomics represents a powerful approach to characterize biochemical networks and to establish functional correlations between genotype and phenotype [10]. Thus, differential proteomic studies on immature and mature fruits have been accomplished on tomato [11,12], grape [13-20], orange [21,22], peach [23-28], strawberry [29], mango [30], papaya $[31,32]$ and apple $[33,34]$ pericarp; some investigations were conducted on selected tissues, such as exocarp or mesocarp [14-18,20-23,25,27,28,30,31]. These studies described a tissuedependent proteome repertoire that present distinctive changes during fruit ripening. As far as apricot, information about its protein composition is very scant; this may be due to different reasons, including the lack of a species-specific database, the low protein content of this fruit and the high concentration of interfering substances (pigments, polysaccharides, polyphenols, etc.).

In this study, we report on the first proteomic characterization of the apricot fruit in relation to ripening. Fruits of P. armeniaca cv. Vesuviana, a regional variety cultivated in the South of Italy and renowned for its peculiar flavor and texture characteristics, were harvested at three maturation stages and proteins extracted. Differently from other species (such as grape, orange, etc.) whose skin and mesocarp tissues can be easily distincted/separated also at the unripe stage, experiments on apricot were performed on the whole fruit. A number of protein species differentially expressed as result of ripening state was identified by means of combined 2-DE and MS procedures. Their putative physiological role is here discussed in relation to the fruit maturation process.

\section{Materials and methods}

\subsection{Fruit material and characterization of corresponding ripening stages}

Five apricot trees (P. armeniaca cv. Vesuviana, Pellecchiella) were grown in a farmland in the surroundings of Naples, Italy, by using standard cultural practices. Based on surface color, fruit samples were harvested at three ripening stages: green (T0), yellow (T1), and deep-orange/red (T2) at 7, 9 and 11 weeks after anthesis, respectively (Table 1); they were selected for uniformity without any damage. For each ripening stage, about 25-30 fruits were harvested and then divided into three biological replicates. After collection, fruits were cleaned, removed of seeds, cut, frozen in liquid $\mathrm{N}_{2}$ and stored at $-80^{\circ} \mathrm{C}$, until used for protein extraction. In parallel, a similar number of fruit samples for maturity stage assessment were not frozen but immediately processed. Flesh firmness, total soluble solids content (SSC), titratable acidity (TA) and total antioxidant capacity (TAC) were measured. Firmness was determined by means of a penetrometer (Effegi, Milan, Italy) bearing a $8 \mathrm{~mm}$ probe, and is expressed as $\mathrm{kg} / 0.5 \mathrm{~cm}^{2}$. Apricot samples for SSC, TA and TAC determinations were homogenized, centrifuged at 15,000 $\times \mathrm{g}$ for $15 \mathrm{~min}$, at $4{ }^{\circ} \mathrm{C}$, and the corresponding supernatants were used. SSC was estimated by means of the Brix degree ( ${ }^{\circ}$ Brix) at $20{ }^{\circ} \mathrm{C}$, as determined by an RFM330 Refractometer (Bellingham Stanley Ltd, UK). TA was determined by titrating a known volume of apricot supernatant with $0.1 \mathrm{~N} \mathrm{NaOH}$ until reaching $\mathrm{pH} 8.1$ with a HI9017 Microprocessor pHmeter (Hanna Instruments) and phenolphthalein as color indicator; it was expressed as content of malic acid per gram of fresh weight of fruits. TAC was determined according to [35] and expressed as micromoles of Trolox equivalents per gram of fresh weight of fruits. Measurements were performed in triplicate on samples from three independent extractions.

\subsection{Protein extraction}

Protein mining was performed by means of a TCA/acetone precipitation, followed by a phenol extraction [36]; a separate 
Table 1 - Pomological features, antioxidant capacity, protein content and protein extraction yield of "Vesuviana" apricots at three ripening stages. Firmness, total soluble solid content (SSC), titratable acidity (TA), total antioxidant capacity (TAC), total protein content (TP) and protein yield after precipitation with TCA/acetone followed by phenol extraction (Y) were determined as described in the experimental section. Measurements were performed in triplicate on samples from three independent samplings. Reported values are mean \pm SEM. FW, fruit weight.

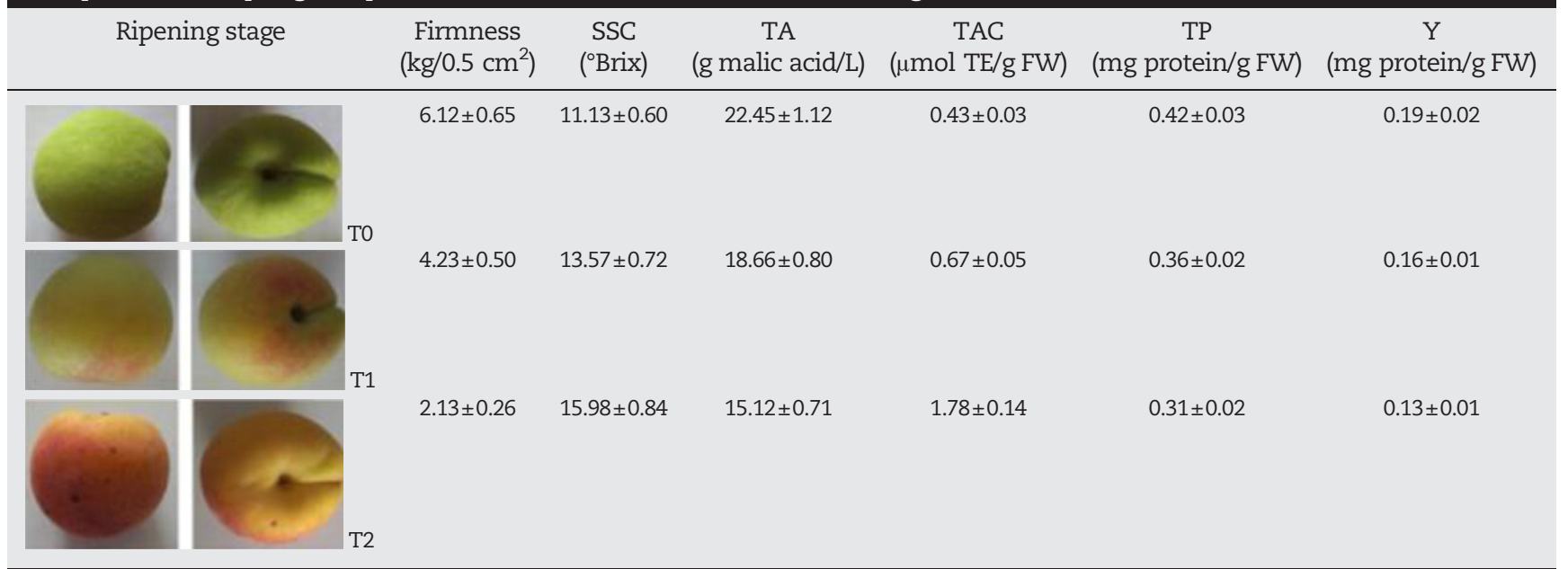

extraction was performed for each biological replicate. Total protein content after the first precipitation step (TP) and final protein extraction yield were determined for each ripening stage. Briefly, frozen fruits were finely powdered in a mortar with liquid $\mathrm{N}_{2}$ and dried under vacuum. Samples containing $2.5 \mathrm{~g}$ of dried tissue were suspended in $10 \mathrm{ml}$ of $10 \% \mathrm{w} / \mathrm{v} \mathrm{TCA} /$ acetone, thoroughly vortexed for $3 \mathrm{~min}$, centrifuged at $16,000 \times g$ for $3 \mathrm{~min}$, at $4{ }^{\circ} \mathrm{C}$, and removed of the supernatant. Samples were extracted with $10 \mathrm{ml}$ of iced $80 \%$ methanol, containing $0.01 \%$ ammonium acetate, and then with $10 \mathrm{ml}$ of iced $80 \%$ acetone, in both cases following the vortexing and centrifugation steps reported above. After air drying at room temperature for $5 \mathrm{~min}$, samples were added with $7.5 \mathrm{~mL}$ of ice-cold extraction buffer (700 mM sucrose), $500 \mathrm{mM}$ Tris-HCl pH 7.5, 50 mM EDTA, $100 \mathrm{mM} \mathrm{KCl,} \%$ w/v $\beta$-mercaptoethanol, $1 \mathrm{mM}$ PMSF, $1 \% \mathrm{w} / \mathrm{v}$ polyvinylpirrolidone, $0.25 \% \mathrm{w} / \mathrm{v}$ CHAPS and then with an equal volume of phenol-saturated-500 mM Tris- $\mathrm{HCl}, \mathrm{pH}$ 8. Samples were extracted in a Waring blender for $5 \mathrm{~min}$ and then centrifuged at $10,000 \times \mathrm{g}$ for $15 \mathrm{~min}$, at $4{ }^{\circ} \mathrm{C}$. Proteins were precipitated from the phenol phase by addition of $5 \mathrm{vol}$ of saturated ammonium acetate in methanol, at $-20^{\circ} \mathrm{C}$, overnight; they were pelletted at $10,000 \times \mathrm{g}$, for $15 \mathrm{~min}$, dried under vacuum, washed at first with iced $0.1 \mathrm{M}$ ammonium acetate in methanol and then twice with cold acetone. During each washing step, vortexing for $3 \mathrm{~min}$, centrifugation at $10,000 \times \mathrm{g}$, at $4{ }^{\circ} \mathrm{C}$, and a brief air drying was performed. Protein concentration was estimated by using the Ramagli and Rodriguez assay [37].

\section{3. $1 \mathrm{D}$ and $2 \mathrm{D}$ electrophoresis}

During SDS-PAGE analysis, protein samples (50 $\mu \mathrm{g})$ were dissolved in loading buffer containing $20 \mathrm{mM}$ DTT and run on a 9-16\% T polyacrylamide gel, which was then stained with colloidal Coomassie G250.

For 2-DE analysis, protein samples were dissolved in IEF buffer (9 M urea, 4\% w/v CHAPS, 0.5\% v/v Triton X-100, 20 mM DTT, $1 \%$ w/v carrier ampholytes pH 3-10, Bio-Rad). IPG strips
(17 cm pH 4-7, ReadyStrip, Bio-Rad) were rehydrated overnight with $300 \mu \mathrm{L}$ of IEF buffer containing $300 \mu \mathrm{g}$ of total proteins. Proteins were focused using a Protean IEF Cell (Bio-Rad) at $12{ }^{\circ} \mathrm{C}$, by applying the following voltages: $250 \mathrm{~V}$ (90 min), $500 \mathrm{~V}$ (90 min), $1000 \mathrm{~V}(180 \mathrm{~min})$ and $8000 \mathrm{~V}$ for a total of $52 \mathrm{kVh}$ [12]. After focusing, the proteins were reduced by incubating the IPG strips with $1 \% \mathrm{w} / \mathrm{v}$ DTT in $10 \mathrm{~mL}$ of equilibration buffer (50 mM Tris- $\mathrm{HCl} \mathrm{pH} \mathrm{8.8,} 6 \mathrm{M}$ urea, 30\% w/v glycerol, $2 \% \mathrm{w} / \mathrm{v}$ SDS and a dash of bromophenol blue) for $15 \mathrm{~min}$, and alkylated with $2.5 \% \mathrm{w} / \mathrm{v}$ iodoacetamide in $10 \mathrm{~mL}$ of equilibration buffer for $15 \mathrm{~min}$. Electrophoresis in the second dimension was carried out on $12 \%$ polyacrylamide gels (180x $240 \times 1 \mathrm{~mm}$ ) with a Protean apparatus (Bio-Rad), using electrophoresis buffer (25 mM Tris-HCl pH 8.3, 1.92 M glycine and 1\% w/v SDS), with $120 \mathrm{~V}$ applied for $12 \mathrm{~h}$, until the dye front reached the bottom of the gel. 2-DE gels were stained with colloidal Coomassie G250. For quantitative analysis, each biological replicate ( 3 in number for each ripening stage) was analyzed in technical triplicate.

\subsection{Image acquisition and analysis}

2D gel images were acquired by using GS-800 a imaging systems (Bio-Rad). Gel digitized images were analyzed by using the PDQuest software (Bio-Rad), which allowed automatic spot detection, landmark identification, spot aligning/ matching within gels and quantification of matched spots; gel manual inspection was performed to correct any error prior to final data analysis. After normalization of the protein spot volume against the spot volume of the entire gel (i.e., of all the protein spots), the percentage volume of each spot was averaged for 9 gels (3 technical replicates of 3 biological replicates). Statistically significant changes in protein abundance were determined by using two sequential data analysis criteria. First, a protein spot had to be present in all gels for each sample to be included in the analysis. Next, statistically significant quantitative changes were determined by using the distribution of fold-change values in the data. Spots were 
determined to be statistically significant if the difference between the average percentage volume of a specific protein spot in the different ripening stages was greater than one standard deviation of the spot volumes for all groups. A two-fold change in normalized spot volumes was then considered as indicative of a significant quantitative variation. For statistical analysis, data were analyzed by using the Statistical Package for Social Science (SPSS) software (IBM SPSS Statistics) through missing value imputation via $\mathrm{K}$-nearest neighbors analysis, followed by logtransformation of the imputed data and comparison of control and treated values to evaluate corresponding variance (ANOVA), with a non-linear mixed-effects model. $P$ values $<0.05$ were considered to be significant.

Differentially expressed proteins from 2D gels were further subjected to a principal component (PC) analysis on a variance/ covariance matrix in order to identify the most relevant proteins. The weight of the coefficients associated with each spot was computed to evaluate which proteins have the greatest impact on the variability explained along the PCs, which are linear combinations of the original variables [25].

\subsection{Protein digestion and MS analysis}

Bands from SDS-PAGE or spots from 2-DE were manually excised from gels, triturated and washed with water. Proteins were in-gel reduced, S-alkylated and digested with trypsin, as previously reported [38]. Protein digests were subjected to a desalting/concentration step on microZipTipC18 pipette tips (Millipore Corp., Bedford, MA, USA) before MALDI-TOF-MS and/or nanoLC-ESI-LIT-MS/MS analysis.

During MALDI-TOF peptide mass fingerprinting (PMF) experiments, peptide mixtures were loaded on the instrument target together with CHCA as matrix, using the dried droplet technique [12]. Samples were analyzed with a Voyager-DE PRO mass spectrometer (Applied Biosystems, USA). Peptide mass spectra were acquired in reflectron mode; internal mass calibration was performed with peptides derived from trypsin autoproteolysis. Data were elaborated using the DataExplorer 5.1 software (Applied Biosystems).

Peptide mixtures were analyzed by nanoLC-ESI-LIT-MS/MS using a LTQ XL mass spectrometer (ThermoFinnigan, USA) equipped with a Proxeon nanospray source connected to an Easy-nanolC (Proxeon, Denmark) [39]. Peptide mixtures were separated on an Easy $\mathrm{C}_{18}$ column $(100 \times 0.075 \mathrm{~mm}, 3 \mu \mathrm{m})$ (Proxeon) using a gradient of acetonitrile containing $0.1 \%$ formic acid in aqueous $0.1 \%$ formic acid; acetonitrile ramped from $5 \%$ to $35 \%$ over $15 \mathrm{~min}$ and from $35 \%$ to $95 \%$ over $2 \mathrm{~min}$, at a flow rate of $300 \mathrm{~nL} / \mathrm{min}$. For shotgun analysis of gel bands from SDS-PAGE, a gradient from $5 \%$ to $35 \%$ over 55 min was used. In both cases, spectra were acquired in the range $\mathrm{m} / \mathrm{z}$ 400-2000. Acquisition was controlled by a data-dependent product ion scanning procedure over the three most abundant ions, enabling dynamic exclusion (repeat count 2 and exclusion duration $1 \mathrm{~min}$ ). The mass isolation window and collision energy were set to $\mathrm{m} / \mathrm{z} 3$ and $35 \%$, respectively.

\subsection{Protein identification}

MASCOT software package version 2.2.06 (Matrix Science, UK) was used to identify proteins present in gel spots and/or bands from an updated plant non-redundant sequence database (NCBI nr 2009/05/03) and/or a Prunus EST database (NCBI nr 2009/05/03). Identified EST entries were associated with corresponding plant proteins by using the BLAST algorithm (http:// blast.ncbi.nlm.nih.gov/). In particular, MALDI-TOF PMF data were searched using a mass tolerance value of $40-80 \mathrm{ppm}$, trypsin as proteolytic enzyme, a missed cleavages maximum value of 2 and Cys carbamidomethylation and Met oxidation as fixed and variable modification, respectively. NanoLC-ESI-LITMS/MS data were searched by using a mass tolerance value of $2 \mathrm{Da}$ for precursor ion and 0.8 Da for MS/MS fragments, trypsin as proteolytic enzyme, a missed cleavages maximum value of 2 , and Cys carbamidomethylation and Met oxidation as fixed and variable modification, respectively. MALDI-TOF PMF candidates with a cumulative MASCOT score $>83$ or nanoLC-ESI-LIT-MS/MS candidates with more than 2 assigned unique peptides with an individual MASCOT score $>25$, both corresponding to $P<0.05$ for a significant identification, were further evaluated by the comparison with their calculated mass and pI values, using the experimental values obtained from SDS-PAGE or 2-DE. Each protein identification was verified manually and a global falsediscovery rate of about $1 \%$ was ascertained by using a reversed protein sequence database [40].

\section{Results and discussion}

\subsection{Physical, chemical and biochemical parameters useful} to obtain a phenotypic definition of the experimental material

The ripening stage of apricot fruit can be defined by physical, chemical and biochemical features that deeply change during the transition from unripe to ripe conditions. In the present work, the classification of the apricot ripening stages was performed according to some selected parameters, such as epicarp ground color, flesh firmness, total soluble solids content, titratable acidity, total antioxidant capacity and protein content (Table 1), which are considered as suitable indicators of the maturation process [35,41-44]. Taken together, these analyses well described the changes that occurred in the three sampled phases of apricot ripening. Resulting data were in good agreement with those from previous studies on this and other apricot cultivars [35,41-44]. In fact, a decrease in flesh firmness was evident during fruit ripening, which was well paralleled with an increase in color index (data not shown), total soluble solids content, and total antioxidant capacity, and with a reduction in titratable acidity and protein content (Table 1); these parameters all concur in defining the overall organoleptic quality of ripening fruit. Ascertained experimental values showed a reduced deviation among the various biological replicates sampled at the same ripening stage, thus indicating a significant physiological similarity between the harvested fruits evaluated in this study.

\subsection{Proteomic analysis of apricot fruits during ripening}

Due to low protein content and high concentration of interfering compounds (Table 1 and data reported on the WorldWideWeb at http://www.thefruitpages.com/contents.shtml), apricot fruits are recalcitrant to proteomic analysis and a protein extraction 
protocol suitable for subsequent electrophoretic analysis needed optimization (data not shown). Best results were obtained by combining two procedures commonly used for difficult plant material, namely TCA/acetone precipitation followed by phenol extraction/ammonium acetate precipitation, which gave protein extracts devoid of interfering substances and sufficiently concentrated for further 2-DE analysis. This approach has been previously used for other fruits [16,17]. The corresponding protein extraction yield for green, yellow and deep-orange/red apricot fruits is reported in Table 1.

At first, protein extracts from fruits at green (T0), yellow (T1) and deep-orange/red (T2) stages were resolved by SDS-PAGE. The resulting gel profile is shown in Fig. 1A; each gel lane was cut into 15 slices, which were then alkylated and digested with trypsin. Peptide digests were then subjected to nanoLC-ESI-LIT-MS/MS experiments, which allowed the total identification of 245 nonredundant gene products (Supporting Information Table S1). Corresponding identification data are reported in Supporting Information Table S2. When identified protein components from SDS-PAGE were compared with those resulting from 2-DE analysis (see below), a general enrichment in polypeptide species having a high molecular mass, a high pI value or a high hydrophobicity was evident (see Supporting Information Table S1 and Table 2 for comparison). Protein distribution between different maturation stages, as ascertained by combined SDSPAGE/nanoLC-ESI-LIT-MS/MS analysis, is reported in Fig. 1B; it highlighted preliminary qualitative differences to be further investigated by dedicated quantitative approaches.

An effective separation of extracted proteins was then obtained by 2-DE, applying a linear 4-7 pH gradient (in the first dimension) and using a $12 \% \mathrm{~T}$ polyacrylamide gel (in the second dimension), which solved components in the range 10-150 kDa. Representative gels for apricot fruits sampled at the different maturation stages are shown in Fig. 2. Average proteomic maps showed $511 \pm 115,554 \pm 80$, and $450 \pm 76$ spots for green, yellow and deep-orange/red stage, respectively. Accordingly, the degree of similarity between the green stage and the others was 77\% (green/yellow) and 71\% (green/deeporange/red). Software-assisted statistical analysis of colloidal Coomassie-stained gels revealed 106 protein spots as differentially represented between various ripening stages $(P<0.05)$. To determine protein identity, these spots were gel-excised, digested and subjected to MALDI-TOF-PMF and/or nanoLCESI-LIT-MS/MS experiments. Due to reduced information on the apricot genome, spot identification by means of MALDITOF peptide mass fingerprinting proved challenging and lead to a positive result only for 34 spots. These difficulties were solved by nanoLC-ESI-LIT-MS/MS analysis, which characterized the remaining 72 spots. In conclusion, 101 spots were identified as protein components mostly from $P$. armeniaca or related species, whereas 5 spots resulted as multiple identifications (Table 2 and Supporting Information Table S3). When results from combined SDS-PAGE/nanoLC-ESI-LIT-MS/MS and 2-DE experiments were compared, common protein species between analyses generally showed a good qualitative trend moving from T0 to T1 and T2 stages (Supporting Information Table S1 and Table 2). However, the higher resolutive power associated with 2-DE analysis allowed to identify specific protein components at a certain ripening stage that, otherwise, were not detected in the corresponding SDS-PAGE/nanoLC-ESILIT-MS/MS experiment. Deregulated gene products identified by 2-DE were categorized into different classes according to Gene Ontology annotation (Fig. 3), thus suggesting that apricot ripening affects polypeptide species involved in various biological processes, with stress/defense proteins being the most represented group, followed by components associated with cell structure, secondary metabolism, energy, general metabolism and other cell functions.

To identify the most relevant proteins among the differentially expressed gene products, a principal component analysis
A

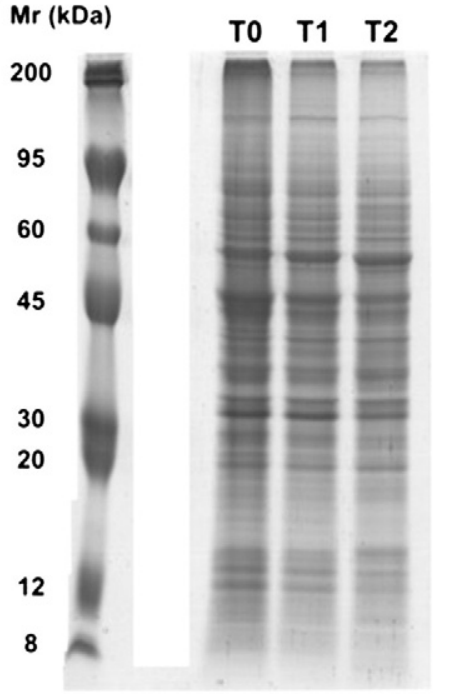

B

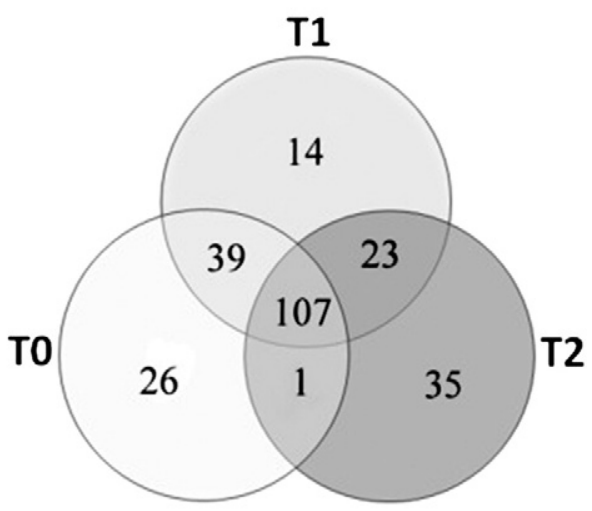

Fig. 1 - Proteomic results from combined SDS-PAGE/nanoLC-ESI-LIT-MS/MS analysis. (A) SDS-PAGE analysis of protein extracts from the apricot fruits shown in Table 1. (B) Distribution of 245 gene products identified by shotgun analysis between the different maturation stages, as shown by the corresponding Venn diagram. 
Table 2 - Protein species showing quantitative changes during apricot ripening as identified by combined 2-DE and MS procedures. Spot number, protein name, EST or proten

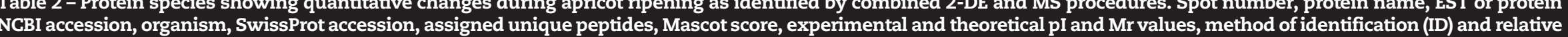
fold change are listed. Increasing/decreasing index (fold change) was calculated as the ratio of spot intensities (relative volumes) for different fruit stages, with respect to the green

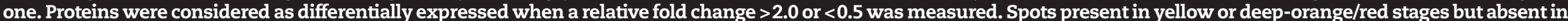
the green stage are reported as +. Spots absent in yellow or deep-orange/red stages but present in the green stage are reported as -. Circles indicate spots assigned with EST entries, which were further associated with specific proteins according to BLAST analysis; the corresponding sequence identity values are reported. Functional grouping was performed according to the Bevan's classification [105]. PMF, MALDI-TOF peptide mass fingerprinting; MSMS, nanoLC-ESI-LIT-MS/MS. Prunus armeniaca, persica, cerasus, avium and dulcis, or Catharanthus roseus, Gevuina avellana, Malus domestica, Vitis vinifera and Zea mays are indicated as P. armeniaca, P. persica, P.cerasus, P.avium and P.dulcis, or C. roseus, G. avellana, M. domestica, V. vinifera and Z. mays, respectively.

\begin{tabular}{|c|c|c|c|c|c|c|c|c|c|c|c|c|c|}
\hline \multirow[t]{2}{*}{ Spot } & \multirow[t]{2}{*}{ Protein name } & \multirow[t]{2}{*}{$\begin{array}{l}\text { EST or } \\
\text { protein NCBI } \\
\text { accession }\end{array}$} & \multirow[t]{2}{*}{ Organism } & \multirow[t]{2}{*}{$\begin{array}{l}\text { Homologous protein - } \\
\text { SwissProt accession } \\
\text { (sequence identity \%) }\end{array}$} & \multirow[t]{2}{*}{$\begin{array}{l}\text { Unique } \\
\text { peptides }\end{array}$} & \multirow[t]{2}{*}{$\begin{array}{l}\text { Mascot } \\
\text { score }\end{array}$} & \multirow[t]{2}{*}{$\begin{array}{l}\text { Theor. } \\
\text { pI }\end{array}$} & \multirow[t]{2}{*}{$\begin{array}{l}\text { Theor. } \\
\text { Mr }\end{array}$} & \multirow[t]{2}{*}{$\begin{array}{c}\text { Exp. } \\
\text { pI }\end{array}$} & \multirow[t]{2}{*}{$\begin{array}{c}\text { Exp. } \\
\mathrm{Mr}\end{array}$} & \multirow[t]{2}{*}{ ID } & \multicolumn{2}{|c|}{$\begin{array}{l}\text { Relative } \\
\text { fold change } \\
\text { (us green) }\end{array}$} \\
\hline & & & & & & & & & & & & Yellow & Deep-orange \\
\hline \multicolumn{14}{|c|}{ Metabolism } \\
\hline 14 & 2-hydroxyacyl-CoA lyase & $209173648^{\circ}$ & P. persica & D2D327 (87) & 7 & 519 & 5.74 & 61,470 & 6.10 & 80,000 & MSMS & 5.1 & 10.0 \\
\hline 28 & Arginase & 63192018 & P. armeniaca & Q1X8N7 (100) & 11 & 125 & 4.60 & 43,353 & 5.78 & 51,700 & PMF & 1.3 & 0.1 \\
\hline 29 & Glutamine synthetase & $89483665^{\circ}$ & P. persica & A9PEY1 (93) & 5 & 297 & 5.95 & 39,448 & 6.30 & 57,300 & MSMS & 3.8 & 11.8 \\
\hline 42 & Acid phosphatase 1 & $30270882^{\circ}$ & P. dulcis & B9H8Y0 (78) & 9 & 157 & 5.00 & 27,759 & 5.06 & 43,100 & PMF & 1.0 & 0.1 \\
\hline 45 & Inorganic pyrophosphatase & $84641872^{\circ}$ & P. persica & Q9M611 (91) & 16 & 176 & 5.64 & 25,931 & 6.24 & 38,200 & PMF & 2.0 & 0.4 \\
\hline 69 & Uridylate kinase & $29953970^{\circ}$ & P. armeniaca & C6T0G4 (86) & 8 & 385 & 5.79 & 22,481 & 5.84 & 31,300 & MSMS & 0.6 & 0.1 \\
\hline 97 & Nucleoside diphosphate kinase 1 & $51503808^{\circ}$ & P. armeniaca & D7LX88 (86) & 10 & 139 & 5.92 & 16,462 & 6.74 & 16,000 & PMF & 0.3 & 0.2 \\
\hline \multicolumn{14}{|c|}{ Energy } \\
\hline 7 & NADP-dependent malic enzyme & $112380989^{\circ}$ & P. cerasus & C8ZK32 (84) & 7 & 444 & 6.16 & 64,799 & 5.64 & 85,200 & MSMS & 2.2 & 2.6 \\
\hline 12 & ATP synthase beta subunit & 194267356 & G. avellana & B4YQ40 (100) & 10 & 101 & 5.14 & 52,950 & 5.72 & 73,200 & PMF & 0.3 & 0.1 \\
\hline 13 & Enolase & $209176176^{\circ}$ & P. persica & D7NHW9 (94) & 15 & 939 & 5.56 & 47,912 & 5.94 & 72,500 & MSMS & 2.7 & 6.4 \\
\hline 20 & Dihydrolipoyl dehydrogenase & $84641323^{\circ}$ & P. persica & I1MRV6 (90) & 6 & 362 & 6.06 & 49,744 & 6.27 & 72,700 & MSMS & 1.0 & 0.2 \\
\hline 25 & Alcohol dehydrogenase 1 & $22487836^{\circ}$ & P. persica & F6K5V5 (97) & 4 & 187 & 5.83 & 41,178 & 5.67 & 62,000 & MSMS & 3.1 & 3.3 \\
\hline 30 & NAD-dependent malate dehydrogenase & $51515746^{\circ}$ & P. armeniaca & E2JE42 (99) & 11 & 742 & 6.60 & 35,497 & 6.33 & 53,300 & MSMS & 3.1 & 5.4 \\
\hline 34 & Isocitric dehydrogenase 5 & $51504432^{\circ}$ & P. armeniaca & B9S0K1 (85) & 7 & 419 & 5.77 & 36,201 & 6.39 & 52,700 & MSMS & 3.1 & 0.4 \\
\hline 43 & Oxygen-evolving enhancer protein 1 & $59932290^{\circ}$ & P. persica & A9PHA9 (93) & 5 & 79 & 5.09 & 26,657 & 5.15 & 42,000 & PMF & 0.2 & 0.1 \\
\hline 44 & Oxygen-evolving enhancer protein 1 & $59932290^{\circ}$ & P. persica & A9PHA9 (93) & 10 & 150 & 5.09 & 26,657 & 5.30 & 42,300 & PMF & 0.8 & - \\
\hline 50 & ATP synthase $24 \mathrm{kDa}$ subunit & $51514717^{\circ}$ & P. armeniaca & D7SW76 (82) & 10 & 142 & 5.30 & 23,999 & 6.80 & 38,200 & PMF & 0.4 & 0.1 \\
\hline 66 & ATP synthase subunit $d$ & $51515825^{\circ}$ & P. armeniaca & A5AY42 (89) & 9 & 399 & 5.09 & 19,454 & 5.23 & 30,000 & MSMS & 0.5 & 0.1 \\
\hline 75 & ATP synthase subunit $d$ & $51515825^{\circ}$ & P. armeniaca & A5AY42 (89) & 34 & 1767 & 5.09 & 19,454 & 5.25 & 27,100 & MSMS & 0.6 & 0.1 \\
\hline \multicolumn{14}{|c|}{ Transcription } \\
\hline 22 & RNA binding protein & $89003232^{\circ}$ & P. persica & D7TGB6 (77) & 5 & 209 & 6.45 & 38,291 & 6.62 & 65,400 & MSMS & 0.2 & 0.2 \\
\hline 82 & Regulator of ribonuclease activity a & $22487660^{\circ}$ & P. persica & D2D305 (89) & 12 & 582 & 5.61 & 17,950 & 5.54 & 20,600 & MSMS & 0.6 & 0.04 \\
\hline 92 & Glycine-rich RNA-binding protein & $51502085^{\circ}$ & P. armeniaca & A5C101 (70) & 2 & 200 & 5.19 & 12,109 & 5.50 & 17,200 & MSMS & 0.7 & 0.1 \\
\hline 94 & Glycine-rich RNA-binding protein & 34851124 & P. avium & Q6YNS1 (100) & 10 & 151 & 7.84 & 17,327 & 6.71 & 19,100 & PMF & 0.2 & 0.1 \\
\hline 95 & Glycine-rich RNA-binding protein & 34851124 & P. avium & Q6YNS1 (100) & 10 & 151 & 7.84 & 17,327 & 6.76 & 19,300 & PMF & 0.5 & 0.1 \\
\hline 99 & Glycine-rich RNA-binding protein & $51502085^{\circ}$ & P. armeniaca & A5C101 (70) & 6 & 382 & 5.19 & 12,109 & 5.46 & 13,600 & MSMS & 0.2 & 0.1 \\
\hline \multicolumn{14}{|c|}{ Protein synthesis } \\
\hline 38 & $\begin{array}{l}\text { Nascent polypeptide-associated } \\
\text { complex subunit alpha-like protein }\end{array}$ & $51502466^{\circ}$ & P. armeniaca & A2PYH3 (89) & 5 & 87 & 4.32 & 22,408 & 4.40 & 40,600 & PMF & 0.6 & 0.1 \\
\hline
\end{tabular}




\begin{tabular}{|c|c|c|c|c|c|c|c|c|c|c|c|c|c|}
\hline 51 & $\begin{array}{l}\text { Nascent polypeptide-associated } \\
\text { complex subunit alpha-like protein }\end{array}$ & $40715286^{\circ}$ & P. armeniaca & A9PA21 (91) & 7 & 589 & 4.32 & 22,408 & 4.44 & 37,000 & MSMS & 0.4 & 0.04 \\
\hline 89 & 60s acidic ribosomal protein & $29957592^{\circ}$ & P. armeniaca & Q8H2B9 (100) & 6 & 469 & 4.26 & 11,414 & 4.30 & 17,300 & MSMS & 0.3 & 0.02 \\
\hline \multicolumn{14}{|c|}{ Protein destination and storage } \\
\hline 6 & Heat shock cognate $70 \mathrm{kDa}$ & 6969976 & M. domestica & Q9M6R1 (100) & 14 & 175 & 5.16 & 71,215 & 5.06 & 84,600 & PMF & 6.1 & 3.7 \\
\hline 61 & Proteasome subunit alpha type & $22482113^{\circ}$ & P. persica & B9RI05 (97) & 9 & 105 & 5.70 & 25,598 & 5.95 & 36,000 & PMF & 1.0 & 0.2 \\
\hline 63 & Proteasome subunit alpha type- 4 & $51917382^{\circ}$ & P. persica & B9RA94 (95) & 12 & 883 & 6.62 & 27,417 & 6.49 & 37,700 & MSMS & 1.5 & 0.2 \\
\hline 70 & Proteasome subunit beta type-1 & $89481856^{\circ}$ & P. persica & D7U7S6 (86) & 3 & 232 & 6.30 & 24,617 & 6.25 & 32,900 & MSMS & 2.5 & 2.6 \\
\hline 88 & $\begin{array}{l}\text { Ubiquitin-conjugating enzyme E2 } \\
\text { variant } 1\end{array}$ & $22489032^{\circ}$ & P. persica & I1JGK4 (97) & 7 & 108 & 6.51 & 16,480 & 6.53 & 20,700 & PMF & 1.1 & 0.1 \\
\hline 96 & Ubiquitin-conjugating enzyme E2 & 5381319 & C. roseus & Q9XHP3 (100) & 5 & 81 & 6.14 & 17,139 & 6.58 & 18,100 & PMF & 1.0 & 0.1 \\
\hline 103 & Subtilisin-like protease & $29957430^{\circ}$ & P. armeniaca & B9R726 (79) & 6 & 481 & 6.38 & 67,571 & 6.43 & 91,600 & MSMS & + & ${ }^{++}$ \\
\hline \multicolumn{14}{|c|}{ Transporters } \\
\hline 56 & Membrane steroid-binding protein & $40715443^{\circ}$ & P. armeniaca & B9I4C2 (73) & 15 & 1034 & 4.42 & 23,289 & 4.55 & 36,800 & MSMS & 0.6 & 0.1 \\
\hline 57 & Membrane steroid-binding protein & $40715443^{\circ}$ & P. armeniaca & B9I4C2 (73) & 15 & 1034 & 4.42 & 23,289 & 4.63 & 36,200 & MSMS & 0.7 & 0.1 \\
\hline 76 & Temperature-induced lipocalin & $22481517^{\circ}$ & P. persica & Q38JC5 (99) & 8 & 442 & 5.60 & 21,463 & 5.71 & 29,800 & MSMS & 0.1 & 0.1 \\
\hline 100 & Acyl CoA binding protein & $29953048^{\circ}$ & P. armeniaca & Q8RVT5 (94) & 4 & 136 & 5.83 & 10,051 & 5.70 & 12,300 & MSMS & 0.1 & 0.3 \\
\hline \multicolumn{14}{|c|}{ Cell structure } \\
\hline 15 & Actin & $22481151^{\circ}$ & P. persica & B6V9Z9 (100) & 2 & 122 & 5.31 & 41,735 & 4.60 & 63,500 & MSMS & 0.4 & 0.1 \\
\hline 21 & Polygalacturonase & $84642133^{\circ}$ & P. persica & Q2LAF3 (100) & 3 & 204 & 6.26 & 38,703 & 6.38 & 65,500 & MSMS & 1.7 & 2.6 \\
\hline 23 & Actin & $22478425^{\circ}$ & P. persica & D5MQE0 (98) & 2 & 125 & 5.46 & 41,365 & 4.86 & 63,000 & MSMS & 2.6 & 3.6 \\
\hline 27 & GRP-like protein 2 & $89486596^{\circ}$ & P. persica & F6HEZ8 (97) & 7 & 365 & 6.01 & 40,846 & 5.72 & 57,500 & MSMS & 0.5 & 0.1 \\
\hline 35 & Annexin D1 & $40715469^{\circ}$ & P. armeniaca & I1KT27 (76) & 17 & 1013 & 5.76 & 36,266 & 6.44 & 48,900 & MSMS & 2.9 & 6.9 \\
\hline 36 & Annexin D1 & $51515150^{\circ}$ & P. armeniaca & I1KT27 (76) & 16 & 223 & 5.76 & 36,266 & 6.47 & 48,800 & PMF & 3.2 & 5.9 \\
\hline 64 & MFP1 attachment factor 1 & $29956109^{\circ}$ & P. armeniaca & F5C0G6 (57) & 7 & 426 & 4.34 & 16,180 & 4.61 & 32,300 & MSMS & 0.2 & 0.1 \\
\hline 65 & MFP1 attachment factor 1 & $29956109^{\circ}$ & P. armeniaca & F5C0G6 (57) & 10 & 685 & 4.34 & 16,180 & 4.71 & 32,400 & MSMS & 0.5 & 0.1 \\
\hline 72 & $\begin{array}{l}\text { Translationally-controlled tumor } \\
\text { protein homolog }\end{array}$ & $22482737^{\circ}$ & P. persica & A5BM68 (91) & 10 & 373 & 4.51 & 19,038 & 4.67 & 27,800 & MSMS & 1.4 & 0.1 \\
\hline 93 & Pectinesterase inhibitor & $29957399^{\circ}$ & P. armeniaca & G7JPZ3 (51) & 7 & 610 & 7.58 & 18,937 & 5.72 & 17,900 & MSMS & 0.1 & - \\
\hline 98 & Profilin & $22485387^{\circ}$ & P. persica & Q8GSL5 (100) & 7 & 473 & 4.67 & 13,929 & 4.62 & 13,600 & MSMS & 0.7 & 0.1 \\
\hline 102 & Actin depolymerizing factor 1 & $22477679^{\circ}$ & P. persica & F6HN87 (89) & 3 & 243 & 5.78 & 16,509 & 5.97 & 19,100 & MSMS & + & ${ }^{++}$ \\
\hline 104 & $\begin{array}{l}\text { Xyloglucan endotransglucosylase/ } \\
\text { hydrolase protein } 32\end{array}$ & $51515054^{\circ}$ & P. armeniaca & I1LR80 (77) & 4 & 222 & 9.53 & 31,928 & 6.26 & 45,600 & MSMS & + & ++ \\
\hline 105 & $\begin{array}{l}\text { Xyloglucan endotransglucosylase/ } \\
\text { hydrolase protein } 32\end{array}$ & $51515054^{\circ}$ & P. armeniaca & I1LR80 (77) & 3 & 182 & 9.53 & 31,928 & 6.46 & 47,000 & MSMS & - & + \\
\hline \multicolumn{14}{|c|}{ Stress response/defense } \\
\hline 10 & Type II SK2 dehydrin & $51514728^{\circ}$ & P. armeniaca & Q30E95 (94) & 5 & 182 & 5.37 & 28,314 & 4.86 & 72,600 & MSMS & 0.8 & 0.1 \\
\hline 11 & Type II SK2 dehydrin & 73762178 & P. persica & Q30E95 (100) & 7 & 76 & 5.37 & 28,314 & 4.92 & 69,600 & PMF & 0.4 & 0.2 \\
\hline 16 & Type II SK2 dehydrin & 73762178 & P. persica & Q30E95 (100) & 7 & 90 & 5.37 & 28,314 & 4.97 & 69,400 & PMF & 1.3 & 0.4 \\
\hline 40 & $\begin{array}{l}\text { Abscisic stress ripening } \\
\text { protein homolog }\end{array}$ & $51504929^{\circ}$ & P. armeniaca & D4Q9L9 (97) & 5 & 312 & 5.64 & 21,240 & 5.34 & 45,000 & MSMS & 0.2 & 0.1 \\
\hline 41 & $\begin{array}{l}\text { Abscisic stress ripening protein } \\
\text { homolog }\end{array}$ & $51503006^{\circ}$ & P. armeniaca & D4Q9L9 (97) & 6 & 558 & 5.64 & 21,240 & 5.71 & 44,000 & MSMS & 0.5 & 0.1 \\
\hline
\end{tabular}




\begin{tabular}{|c|c|c|c|c|c|c|c|c|c|c|c|c|c|}
\hline 17 & S-adenosylmethionine synthetase & $22481305^{\circ}$ & P. persica & Q9AT56 (95) & 6 & 82 & 5.34 & 43,150 & 5.72 & 65,800 & PMF & 0.2 & 0.1 \\
\hline 18 & Methionine adenosyltransferase 2 & $22484113^{\circ}$ & P. persica & Q9AT55 (98) & 8 & 122 & 5.50 & 43,135 & 5.84 & 64,400 & PMF & 1.0 & 0.1 \\
\hline 19 & IAA-amino acid hydrolase & $29952725^{\circ}$ & P. armeniaca & F6HGX2 (74) & 6 & 198 & 6.12 & 45,572 & 5.93 & 68,000 & MSMS & 1.0 & 0.2 \\
\hline 24 & Quinone oxidoreductase-like protein & $23033107^{\circ}$ & P. persica & Q941I0 (83) & 5 & 421 & 8.46 & 40,986 & 4.88 & 53,200 & MSMS & 2.0 & 36.2 \\
\hline 26 & Methionine adenosyltransferase 2 & $22484113^{\circ}$ & P. persica & Q9AT55 (99) & 10 & 149 & 5.50 & 43,135 & 5.86 & 64,600 & PMF & 2.4 & 0.3 \\
\hline 31 & Beta-cyanoalanine synthase 1 & $112381831^{\circ}$ & P. cerasus & Q1KLZ2 (87) & 10 & 499 & 7.61 & 40,883 & 6.45 & 55,800 & MSMS & 0.8 & 0.1 \\
\hline 32 & Quinone oxidoreductase-like protein & $23033107^{\circ}$ & P. persica & Q941I0 (83) & 16 & 1050 & 8.46 & 40,986 & 5.54 & 53,900 & MSMS & 1.3 & 3.0 \\
\hline 37 & Isoflavone reductase related protein & $115594788^{\circ}$ & P. persica & 081355 (89) & 6 & 523 & 6.02 & 33,822 & 6.67 & 48,800 & MSMS & 0.6 & 7.6 \\
\hline 67 & Chalcone flavonone isomerase & $89479121^{\circ}$ & P. persica & F2VR46 (98) & 7 & 441 & 5.26 & 23,605 & 5.66 & 31,200 & MSMS & 0.6 & 0.2 \\
\hline 101 & $\begin{array}{l}\text { 1-aminocyclopropane-1-carboxylate } \\
\text { oxidase }\end{array}$ & $84641159^{\circ}$ & P. persica & Q9LRC0 (99) & 17 & 1098 & 5.28 & 36,242 & 5.26 & 55,200 & MSMS & + & ${ }^{++}$ \\
\hline \multicolumn{14}{|c|}{ Unclear classification } \\
\hline 33 & Thiosulfate sulfurtransferase & $115593083^{\circ}$ & P. persica & I1KTY9 (91) & 12 & 700 & 6.57 & 41,547 & 5.71 & 48,600 & MSMS & 0.2 & 0.1 \\
\hline 59 & Stem-specific protein TSJT1 & $29956597^{\circ}$ & P. armeniaca & I1MR83 (92) & 2 & 153 & 6.06 & 29,530 & 5.58 & 35,000 & MSMS & 3.7 & 4.3 \\
\hline 71 & Elicitor responsive gene 3 & $29955053^{\circ}$ & P. armeniaca & Q0JBH9 (43) & 3 & 266 & 3.94 & 15,666 & 4.23 & 27,500 & MSMS & 0.6 & 0.1 \\
\hline \multicolumn{14}{|c|}{ Unclassified } \\
\hline 54 & Uncharacterized protein & $51503356^{\circ}$ & P. armeniaca & D7SK38 (62) & 7 & 122 & 4.95 & 18,727 & 4.75 & 42,100 & PMF & 2.1 & 0.1 \\
\hline 55 & Uncharacterized protein & $51501606^{\circ}$ & P. armeniaca & D9IZZ9 (62) & 6 & 84 & 5.48 & 18,914 & 4.75 & 39,800 & PMF & 0.3 & 0.1 \\
\hline 58 & Unnamed protein product & 297744880 & V. vinifera & F6I216 (97) & 6 & 85 & 9.64 & 68,484 & 4.71 & 37,900 & PMF & 1.7 & 9.1 \\
\hline 86 & Uncharacterized protein & $29952094^{\circ}$ & P. armeniaca & F6HZZ7 (40) & 10 & 621 & 5.75 & 52,888 & 6.32 & 23,600 & MSMS & 0.3 & 0.1 \\
\hline 90 & Uncharacterized protein & $29955823^{\circ}$ & P. armeniaca & I1J728 (82) & 3 & 141 & 5.94 & 18,415 & 5.19 & 17,500 & MSMS & 0.4 & - \\
\hline \multicolumn{14}{|c|}{ Multiple identifications } \\
\hline \multirow[t]{2}{*}{1} & Cell division cycle protein & $89492273^{\circ}$ & P. persica & I1LL20 (98) & 5 & 311 & 5.07 & 90,416 & 5.72 & 97,400 & MSMS & 0.2 & 0.1 \\
\hline & Aconitate hydratase & $51515268^{\circ}$ & P. armeniaca & G3BMW4 (96) & 7 & 307 & 6.66 & 110,056 & & & & & \\
\hline \multirow[t]{2}{*}{39} & Uncharacterized protein & $40714873^{\circ}$ & P. armeniaca & B7FH09 (64) & 6 & 322 & 4.93 & 23,797 & 5.05 & 45,700 & MSMS & 0.5 & 0.1 \\
\hline & Inorganic pyrophosphatase & $46547289^{\circ}$ & P. persica & B9RX33 (90) & 9 & 554 & 4.61 & 20,470 & & & & & \\
\hline \multirow[t]{2}{*}{47} & Predicted protein & $22488713^{\circ}$ & P. persica & A5C3G7 (87) & 7 & 603 & 6.18 & 29,403 & 6.49 & 39,800 & MSMS & 1.3 & 0.4 \\
\hline & Glyoxalase II & $115591916^{\circ}$ & P. persica & B9S301 (88) & 10 & 559 & 4.60 & 43,353 & & & & & \\
\hline \multirow[t]{2}{*}{68} & DSBA oxidoreductase & $84637780^{\circ}$ & P. persica & D7SMN4 (73) & 5 & 223 & 8.62 & 28,035 & 5.77 & 32,100 & MSMS & 0.2 & 0.1 \\
\hline & Benzoquinone reductase & 5031275 & P. armeniaca & Q9XH74 (100) & 2 & 143 & 6.97 & 21,756 & & & & & \\
\hline \multirow[t]{2}{*}{87} & Uncharacterized protein & $29954933^{\circ}$ & P. armeniaca & I1LB31 (78) & 5 & 284 & 8.64 & 21,325 & 6.72 & 23,300 & MSMS & 0.7 & 6.0 \\
\hline & Uncharacterized protein & $29952094^{\circ}$ & P. armeniaca & F6HZZ7 (40) & 9 & 553 & 5.75 & 52,888 & & & & & \\
\hline
\end{tabular}



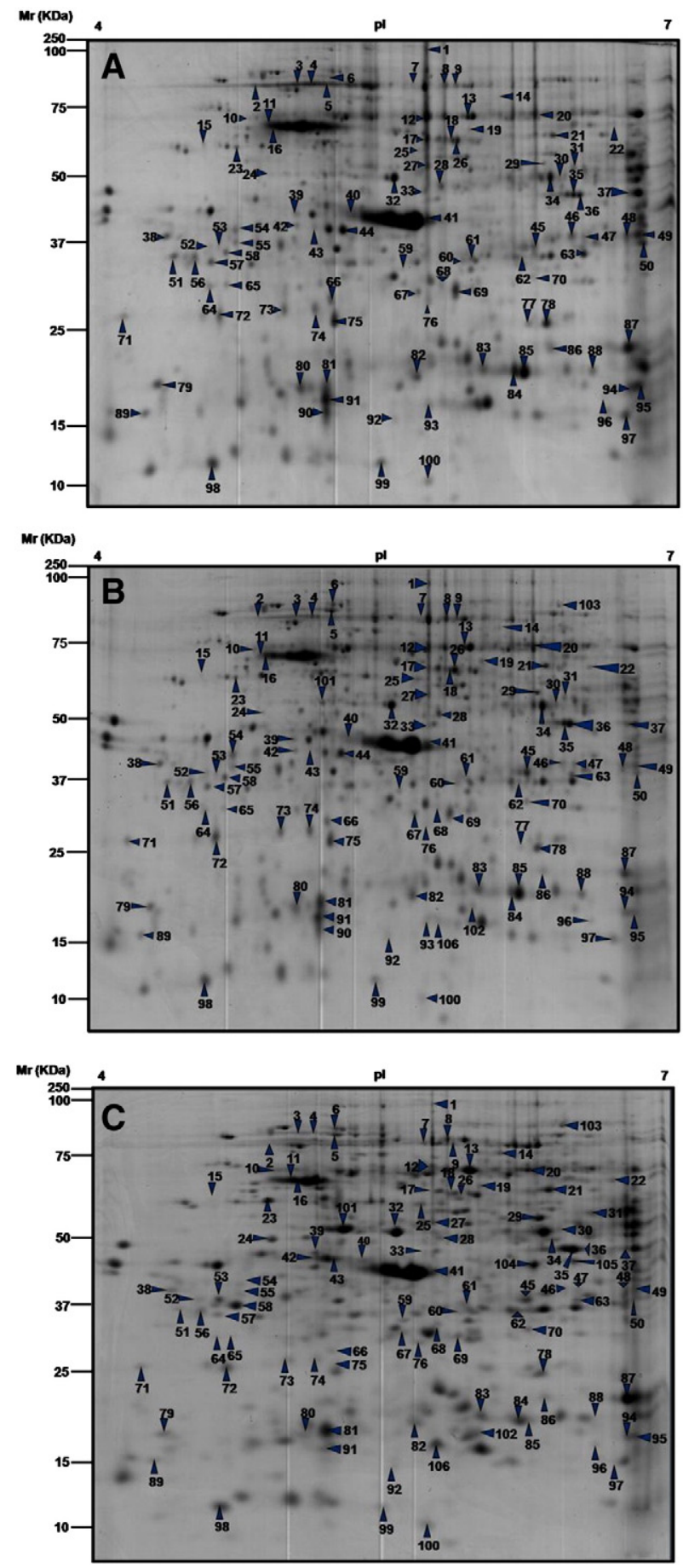

Fig. 2 - Two-dimensional electrophoresis maps of total flesh proteins from apricot fruits at green (T0) (A), yellow (T1) (B) and deep-orange/red (T2) (C) stages. Protein spots differentially expressed are numbered. Proteins were separated over the pI range 4-7 in the first dimension and on $12 \%$ SDS-polyacrylamide gels in the second dimension. Gels were stained with colloidal Coomassie G250.

was also performed on a variance/covariance matrix (Fig. 4 and Supporting Information Table S4). The first component (PC1) explained the greater part $(90.93 \%)$ of the total variance and was able to separate $\mathrm{T} 2$ and $\mathrm{T} 0 / \mathrm{T} 1$; on the other hand, T0 and T1 were distinguished along PC2, thus explaining a small part $(7.32 \%)$ of the total variance. The most relevant protein species (coeff. $>0.1$ or $<-0.1$ ) were identified for the first two PCs. Along PC1, polygalacturonase, major allergen Pru p1, 60S acidic ribosomal protein, allergen Pru p1.06B, ubiquiting conjugating enzyme E2, nucleoside diphosphate kinase, profilin and acyl CoA-binding protein gene products (spots 21, 85,89, 91, 96, 97, 98, and 99, respectively) distinguished T2, while type II SK2 dehydrin, ATP synthase $\beta$ subunit, $\beta$-cyanoalanine synthase and acid phosphatase components (spots 11, 12, 31 and 42, respectively) were indicative of $\mathrm{TO}$ and $\mathrm{T} 1$. Along PC2 that discriminated T0 and T1, heat shock cognate $70 \mathrm{kDa}$, ATP synthase $\beta$ subunit, enolase, actin, NAD-dependent malate dehydrogenase, acid phosphatase, oxygen-evolving enhancer protein 1, pectinesterase inhibitor and nucleoside diphosphate kinase gene products (spots 6, 12, 13, 23, 30, 42, 43, 93 and 97, respectively) were indicative of T0, while type II SK2 dehydrin, IAA-amino acid hydrolase, polylacturonase, abscisic stress ripening protein homolog, major allergen Pru p1, 60S acidic ribosomal protein, allergen Pru p1.06B and profilin components (spots 11, 19, 21, 41, 85, 89, 91 and 98, respectively) characterized T1.

\subsection{Proteins associated with hormone and cell wall metabolism}

In climacteric fruits, ripening is preceded by a characteristic burst in ethylene levels, which triggers metabolic/structural changes associated with the maturation process, including loss of mesocarp firmness. Pulp softening depends on the alteration of the cell wall structural properties, with massive depolymerization/solubilization of cell wall components. Although some processes appear to be common to most species, many others seem to be peculiar, involving the activation of different sets of cell wall-modifying enzymes [45].

In accordance with the pivotal role of ethylene in apricot ripening, we identified four enzyme species involved in metabolism of this phytohormone as progressively deregulated, namely 1-aminocyclopropane-1-carboxylate (ACC) oxidase (ACO, spot 101), two S-adenosylmethionine (SAM) synthetases (MAT1, spot 17; MAT2, spot 18 and 26) and $\beta$-cyanoalanine synthase (CYSC1, spot 31); the first one showed increased abundance during maturation, while the remaining ones showed an opposite trend. ACO is responsible for direct conversion of ACC into ethylene; in peach, its transcript is the most largely up-regulated during the transition from the pre-climateric to the climateric phase [46]. By producing $\beta$-cyanoalanine, on the other hand, CYSC1 detoxifies cyanidric acid resulting as side product from ACC oxidation. Finally, S-adenosylmethionine synthetases catalyze the first reaction of the ethylene biosynthesis pathway yielding SAM, but are also involved in other biochemical events since this product generally acts as a main methyl group donor [9]. Quantitative levels of these four enzymes during fruit ripening were in good agreement with the corresponding changes of ethylene concentration, as measured in other apricot cultivars [42,47], and showed a significant similarity with what already reported for these proteins in peach mesocarp in the course of maturation and post-harvest storage $[25,27,28]$.

In some fleshy fruits, levels of phytohormone indole-3acetic acid (IAA) decline towards the onset of ripening [48-50]; 


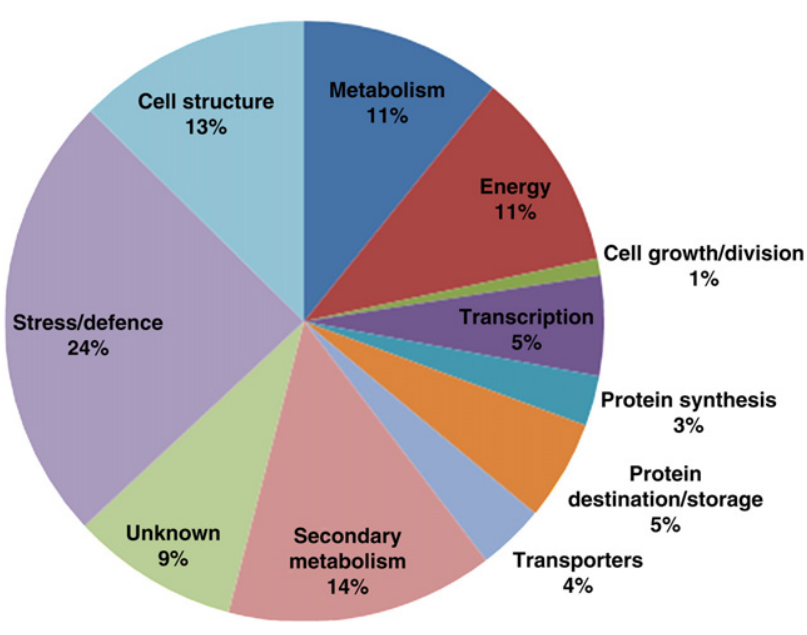

Fig. 3 - Functional distribution of 101 deregulated gene products as identified by 2-DE and MS procedures. Identified protein species were categorized into different classes according to Gene Ontology annotation and Bevan's classification [105]. Percentual discrepancies with respect to categorization present in Table 2 were due to the eventual attribution of individual protein entries into multiple functional classes.

however, the mechanisms by which these low concentrations are achieved during fruit maturation remain elusive. Transcriptomic studies on grape and tomato fruit maturation, combined with quantitative conjugated IAA-amino acid and IAA assays, demonstrated that an increased expression of IAA-amido synthetases coincides with low and high concentrations of IAA and IAA-amino acids, respectively [48,51]. In this context, we observed a progressive quantitative reduction of a IAA-amino acid hydrolase species (spot 19) during apricot maturation. It is conceivable that this protein decrease also contributes to lower free IAA levels during ripening.

Four enzyme species involved in cell wall metabolism showed variable quantitative levels during apricot maturation, namely endopolygalacturonase (PG, spot 21), xyloglucan endotransglucosilase/hydrolase protein 32 (XTH32) (spot 104 and 105), pectinesterase inhibitor (PEI, spot 93) and GRP-like protein 2 (GRPL2, spot 27). EndoPGs, in concert with

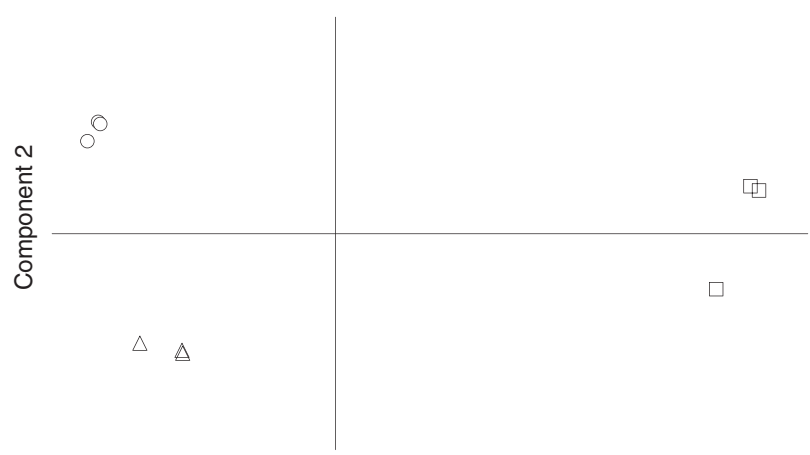

Component 1

Fig. 4 - Scatter plot of the first two principal components visualizing how the apricot samples can be separated along a time gradient of maturation. Circles, triangles and squares indicate T0, T1 and T2 biological replicates, respectively. pectinesterases, are responsible for massive hydrolysis and solubilization of polyuronides during peach ripening, determining fruit softening $[25,28,52,53]$. Remarkably, the EndoPG isoform we identified as up-regulated during apricot maturation is identical to that whose over-expression has been already associated with peach mesocarp softening by proteomic $[25,28]$ and transcriptomic investigations [52]. The latter study also identified PEIs as class of ripen-dependent downregulated genes [52]. By confirming this investigation for another climacteric fruit, our data on PEI expand this observation at the proteomic level. Relative contribution of different polysaccharide depolymerization processes in fruit softening, and hence of corresponding enzymes, is still unclear and may differ from species to species; for instance, preferential xyloglucan rather than polyuronide degradation occur in tomato ripening [54]. XTHs have been associated with ripening and softening of different fruits, where they can either determine cell wall integrity maintenance or weakening; in this context, opposite quantitative trends for XTHs have been observed in tomato [54] and grape skin during ripening $[14,15,17]$, respectively. Data reported in this study prove a XTH32 component overproduction even at the fruit yellow stage, thus pointing to a concerted remodeling of xyloglucans and polyuronides through apricot ripening. GRPL2 is thought to have a role, not yet clarified, in the biosynthesis of hemicellulosic polysaccharides present in the cell wall $[55,56]$. In agreement with this hypothesis, protein species concentration decreased throughout apricot maturation.

Some gene products whose function is related to cytoskeleton organization were also identified as deregulated, including actin isoforms (ACT7 and CACT1, spots 15 and 23), profilin (PF, spot 98), actin depolymerising factor 1 (ADF, spot 102), annexin D1 (ANX2, spot 35 and 36) and translationally-controlled tumor protein homolog protein (TCTP, spot 72) species that, depending on their nature, showed reduced and augmented levels, respectively. Their quantitative changes during apricot ripening suggested the occurrence of regulative processes that affect 
cytoskeletal and membrane cell proteins, thus modulating cell shape, cell division, organelle movement, and extension growth [57]. In plant cells, responses to hormones or environmental stresses, along with cell morphogenesis pathways, induce signaling cascades that correlate with the rearrangement/ turnover of actin-based structures [58-60]. In this context, ADF and profilin are small actin-binding proteins that control actin dynamics [61,62]; TCTP also interacts with cytoskeletal actin and plays essential roles in cell protection against various stresses [63,64]. Annexins are $\mathrm{Ca}^{2+}$-dependent, membranebinding proteins that fasten and possibly hold together biological structures [65]; their expression is under developmental and environmental control. In tomato, it has been shown that ANXs have a F-actin binding activity [66], but information on their role in fruit maturation is quite limited. Since ANXs may form/regulate $\mathrm{Ca}^{2+}$ channels in plasma and internal membranes, thus increasing vacuole volume and cell wall/plasma membrane surface, they have been suggested to play a role in cell expansion during fruit growth [67]. In conclusion, we identified protein-specific quantitative changes that were in agreement with data already reported on whole peach [26] and strawberry [29] fruits, or grape skin [14,15] during ripening and softening, thus corroborating the hypothesis that these variations may be related to a progressive cytoskeletal disorganization, concomitant to that of cellular wall.

\subsection{Proteins associated with response to different stresses}

Ripening of climacteric fruits involves a marked increase of respiration and, consequently, alteration of the redox homeostasis of cells, with reactive oxygen species (ROS) accumulation, which in turn determines lipid peroxidation, protein denaturation and metabolism deterioration [24,68], to achieve a final degradation state functional to seed release. ROS accumulation is a gradual phenomenon, modulated by a battery of antioxidant metabolites and cellular antioxidant enzymes, whose concentration varies during the different stages of ripening [24]. In two apricot genotypes, for example, accumulation of total phenolics and vitamin $\mathrm{C}$, total radical scavenging activity and both water- and lipid-soluble antioxidant capacities demonstrated the increase of antioxidant compounds/capacities during fruit maturation [44]; these data well paralleled with the TAC results reported here (Table 1). In this study, we measured a variable expression of antioxidant enzyme gene products; in particular, a progressive induction of cytosolic [Cu-Zn]-superoxide dismutase (SOD, spot 106) and L-ascorbate peroxidase (APX, spot 62) species, and a concomitant reduction of chloroplastic dehydroascorbate reductase (DHR, spot 60), glutathione peroxidase 1 (GPX1, spot 73 and 74) and phospholipid hydroperoxide glutathione peroxidase (GPX2, spot 77 and 78) species was observed. In the case of $[\mathrm{Cu}-\mathrm{Zn}]-\mathrm{SOD}$, our results well correlated with that from previous studies on whole tomato fruit [12], different grape tissues [17], papaya mesocarp [32] and mango mesocarp [30], where a general increase in SODs, and specifically in [Cu$\mathrm{Zn}$ ]-isoform, content was associated with fruit maturation. All these studies provided contrasting results with other reports on SOD activities during fruit ripening where, as in the case of tomato, the apex was detected at the beginning of maturation process to decline thereafter [68] or, as in the case of peach, no statistically significant changes were measured in the transition from immature to mature stage [69], although a modification of the protein isozyme pattern was observed. On the other hand, apricot levels of cytosolic APX species during fruit maturation showed a quantitative trend similar to what already observed in whole tomato [11,12], strawberry [29] and grape [13] fruits; conversely, constant amounts of APX were measured in mesocarp during peach softening $[25,28]$. APX (together with catalase) is the main enzyme responsible for $\mathrm{H}_{2} \mathrm{O}_{2}$ removal in plant subcellular compartments, where it occurs as multiple isoforms [12]. Another enzyme of the ascorbate-glutathione cycle was also identified in this study, namely chloroplastic DHR, whose levels decreased through the ripening stages, as already observed in tomato [12]; this trend was also observed for some GPX1 and GPX2 species and was the opposite to that measured for these enzymes in grape skin [15] and peach mesocarp [28]. In plants, GPXs are members of the peroxiredoxin family that use thioredoxin instead of glutathione as reductant. They are probably a part of an alternative pathway to scavenge phospholipid hydroperoxides, thereby protecting membranes; their levels may increase or decrease in isozyme-specifc fashion in response to different stresses [70]. Since it has been shown that significant differences may be observed among species in the fruit antioxidant profile [71], it is tempting to speculate that the levels of these antioxidant enzymes are modulated according to the pool of antioxidant metabolites in a species-specific fashion.

Heat shock proteins (HSPs) play a fundamental role as protein and membrane stabilizers during fruit ripening/post harvesting and a marked increase, particularly of small HSPs, have been reported in different fruit tissues [11,14,25-29]. Their chaperone activity could help the fruit cope with stressful conditions that would result in an increase of protein misfolding [24]. In this study, no small HSPs were identified as deregulated proteins, but rather a $70 \mathrm{kDa}$ heat shock cognate protein species (HSC70, spot 6) that was induced, with a peak at the apricot yellow ripening stage. Heat shock 70 protein family comprises both heat-inducible as well as constitutivelyexpressed members [72], whose levels have been shown to increase upon different abiotic/biotic stresses [72,73] or to change in a isoform-specific fashion in whole grape [13], tomato [12] and strawberry [29] fruits or papaya mesocarp [31,32] during ripening.

A number of proteins involved in plant response to abiotic stresses, namely abscisic acid stress ripening homolog (ASR, spot 40 and 41), type II SK2 dehydrin (DH, spot 10, 11 and 16) and universal stress protein (USP, spot 83) species and two glycine-rich RNA-binding protein isoforms (GR1, spot 92 and 99; GR2, spot 94 and 95), were observed as down-represented during apricot maturation. ASRs are a family of small hydrophilic proteins having chaperone-like or transcription control activities [74]; recently, they have been demonstrated being able to scavenge ROS in vitro [75]. Dehydrins protect cell compartments from dehydration and are induced by different stress. USPs assist tomato fruit development and ripening against a variety of stresses [11], and may be induced and mediated by ethylene during Arabidopsis and rice ripening. Glycine-rich RNA-binding proteins are implicated in plant response to environmental stresses, particularly salinity and drought [76]. They seem to have a RNA chaperone activity and 
could help in maintaining redox homeostasis during ripening, since it has been reported that catalase and peroxidase activities are affected by mitochondrial-localized GRs expression under temperature stress [77]. When specific stress-response proteins were evaluated, our quantitative data were generally in good agreement with previous results on maturation or postharvesting in whole tomato [11] and peach [26] fruits, peach mesocarp [25,28], and grape skin [15,17], although some subtle differences were also observed.

Various proteins related to plant response to biotic stress were also differentially represented during apricot ripening; among that, proteins having a direct protective function on fruits from pathogen attack, such as acidic endochitinase species (CHIT, spot 52 and 53), which were strongly up-regulated at the ripen stage, as already observed in papaya mesocarp [31] and different grape tissues $[14,15,17]$. In plants, endochitinases play a major role in degrading chitin, a structural component of the fungal cell wall; they occur as many isoforms and cause food allergies, being responsible of the latex-fruit cross sensitivity syndrome [78]. Other allergens, such as sequence-related major allergen Pru p1 (spots 84 and 85), Pru p1.06B (spots 81 and 91) and Pru ar1 (spots 79 and 80) species, generally showed reduced levels during ripening, except one spot. These pathogenesisrelated (PR) proteins are present at a variable level in different Rosaceae tissues upon pathogen fruit infection, ripening and softening [11,15,20,25-29,79]. They are the causative agents of the birch pollen-related food allergy. Although differences in antigens concentration have been reported among peach cultivars [80], no data are available on apricot; this information should be very helpful for breeding of hypoallergenic landraces. A decreasing concentration trend was also observed for some remorin isoforms (REM, spot 46, 48 and 49), which are functionally associated with plant defense, probably having a regulatory role [81].

Protein species involved in the biosynthesis of secondary metabolites were also included in this paragraph, due to the importance of these compounds in adaptative responses. They included polyphenol oxidase (PPO, spots 2-5, 8 and 9), which catalyzes the $\mathrm{O}_{2}$-dependent oxidation of monophenols and o-diphenols to o-quinones, molecules involved in browning reactions as a consequence of pathogen infection, wounding and organ senescence [82], and isoflavone reductase related protein (IFR, spot 37). During apricot maturation, PPO and IFR abundance decreased and increased, respectively, as already observed in different peach [26,28], strawberry [29] and grape $[13,15,17,20]$ tissues. The first gene product is highly represented in immature fruits and is silenced at early stages of ripening, when fleshy fruits start becoming palatable. Although isoflavonoids occur almost exclusively in legumes, IFR related proteins have also been isolated from non-leguminous plants, but their function is still undetermined [83]. Since they contain a $\mathrm{NAD}(\mathrm{P}) \mathrm{H}$-binding domain, they have been proposed to function in the antioxidant response [84]. Recently, it has been shown that their overexpression in rice confers plant tolerance to ROS [85]; data reported here on apricot should represent a first indication that IFR may also play a similar role in fleshy fruit maturation. Additional deregulated gene products included quinone oxidoreductase-like protein (QO, spot 24 and 32) and chalcone flavone isomerase (CFI, spot 67) species, which were up- or down-represented, respectively, during apricot ripening. An increase of $\mathrm{QO}$ was also shown to occur throughout maturation stages in peach mesocarp [28] and whole strawbwerry fruit [29]. This protein has been associated with the biosynthesis of furaneol, a main component of different fruit flavors [86]. Since furaneol occurs also in other fruits, additional studies are necessary to unveil the occurrence of this or related compounds in apricot. On the other hand, CFI catalyzes the conversion of bicyclic chalcones into tricyclic flavanones in the flavonoid biosynthetic pathway, contributing to fruit quality traits.

\subsection{Proteins associated with carbon metabolism and energy production}

During development and ripening of climateric fruits, organic (malic and citric) acids concentration varies in the vacuoles of mesocarp cells and contributes to organoleptic properties [12]. Early accumulation therein is followed by malate release and degradation at the onset of ripening, to fuel the respiratory climax. In this context, phosphoenolpyruvate (PEP) carboxylase and NAD-dependent malate dehydrogenase in the cytosol are the key enzymes for malic acid biosynthesis, while mitochondrial NAD-dependent malate dehydrogenase and cytosolic NADP-dependent malic enzyme are responsible for malate oxidation $[87,88]$. However, other metabolic pathways (gluconeogenesis, fermenative production of ethanol, amino acid and secondary metabolite biosynthes) include malic acid as a carbon source, contributing to complicate its metabolism in fruits, and malate concentration profiles have been reported varying to a high degree among species [88]. Vesuviana apricot is much appreciated for its reduced acidity and elevated content of sugars in the ripe stage $[35,41]$ (see Table 1 ). In this study, the concentration of NAD-dependent malate dehydrogenase (MDH, spot 30) and NADP-dependent malic enzyme (NADP-ME, spot 7) species showed a progressive over-representation during ripening. Augmented MDH and NADP-ME levels should sustain the fruit respiratory climax mentioned above and may justify the reduced acidity of this apricot cultivar (Table 1), respectively. This trend showed some analogies with tomato, grape skin and papaya mesocarp $[12,15,17,31]$ but a poor correlation with other fruit tissues $[13,25,30]$. Mitochondrial NAD-dependent isocitrate dehydrogenase (IDH5, spot 34) concentration also increased in the transition from green to yellow stage, while decreasing thereafter, fairly in accordance with the burst in climacteric fruit respiration at the onset of ripening [27,42]. Different studies have reported an increase of carbon flux through glycolysis in the course of fruit maturation [89] and an increase of glycolytic enzymes in various tissues at the transcript and protein level $[17,27,52,90]$. In order to fuel the characteristic malate changes occurring during ripening, sustained sucrose degradation through glycolysis is necessary. Enolase is considered a key enzyme for PEP generation, since it catalyzes the last step of the glycolytic pathway. In agreement with this scenario, enolase (ENO, spot 13) levels strongly increased during apricot ripening. While contrasting data have been reported as far as ENO mRNA accumulation in tomato ripening [89], our proteomic data are fully consistent with a primary role of the glycolytic pathway in the generation of carbon skeletons (PEP) to fuel the respiratory climax present in ripe apricot [42]. When glycolysis rates overcome respiration 
and generate excess of cytosolic pyruvate, fermentative production of ethanol may also occur, a phenomenon that takes place under aerobic conditions too. In this context, worth noting was the marked increase of alcohol dehydrogenase species (ADH, spot 25) present during apricot ripening, likewise other fruit tissues $[13,29,30]$. It has been also suggested that $A D H$ activity is important for the generation of volatile compounds determining fruit taste and aroma; in this respect, the high ADH levels measured in the ripe Vesuviana cultivar may contribute to its peculiar fragrance characteristics.

On the other hand, mitochondria and chloroplasts of ripen fruits become increasingly damaged or non-functional [12,24, 91-93]. These conditions would lead to cell energy impairment at final maturation stage. Our findings support this statement, since we found a down-regulation of mitochondrial ATP synthase $24 \mathrm{kDa}$ (spot 50) and d (spot 66 and 75) subunits, dihydrolipoyl dehydrogenase (spot 20) as well as of chloroplast ATP synthase subunit $\beta$ (spot 12) and oxygenevolving enhancer protein 1 (spot 43 and 44) species. A similar quantitative trend for specific components has been already described in other fruit tissues [11-13,17,20,28,30,34]. Being involved in the photosynthetic-based NADPH generation or core components of the photosynthetic complexes [94,95], their quantitative decrease would impair the capacity of the ripe fruit cells to generate energy and reducing power. This would lead to the synthesis of NADPH and ATP by cytosolic resident metabolic pathways, such as the oxidative pentose phosphate, glycolytic and fermentative ones (see above) [90].

\subsection{Proteins associated with amino acid and nucleic acid metabolism}

Protein levels are higher in some immature fruits and decline at the onset of ripening to produce amino acids for increased metabolism [13,27] (Table 1). By using a metabolomic approach, it has been proposed that peach amino acids are used to support respiration and as substrates in phenylpropanoid and lignin pathways for endocarp hardening of stone fruits [96]. Accordingly, enzyme species assisting protein biosynthesis, such as nascent polypeptide-associated complex subunit $\alpha$ (spots 38 and 51) [15] and 60S acidic ribosomal protein (spot 89), or involved in their degradation, such as subtilisin-like protease (spot 103), were down- and over-represented during apricot ripening, respectively. Proteins of the ubiquitin-proteasome machinery also varied their levels, i.e. ubiquitin-conjugating enzyme E2 isoforms (spots 88 and 96) and proteasome subunits $\alpha$ (spots 61 and 63) and $\beta$ (spot 70) species, thus confirming previous evidences on their involvement in stress response and fruit maturation $[26,30]$. Their quantitative levels have to be further investigated by dedicated redox proteomic studies relating their concentration to the corresponding ROS and carbonylated protein levels, thus elucidating the role of the ubiquitin-proteasome machinery in the fruit ripeningassociated oxidative stress. On the other hand, a glutamine synthetase gene product (GS, spot 29) showed a strong increase during apricot ripening, as already observed in peach mesocarp and whole strawberry fruit $[28,29]$. This enzyme is central in nitrogen metabolism since it plays a pivotal role in nitrate/ ammonia assimilation and ammonia reassimilation during photorespiration [97]. Although information on this protein are scarce, the observed trend suggests that its over-expression may assist reassimilation of nitrogen produced by protein degradation and amino acid metabolism. Finally, arginase (spot 28) showed a peak at yellow stage and then was down-represented. This enzyme assists arginine hydrolysis to L-ornithine, a precursor of polyamines, which are known to play a role in plant development, defense and NO generation [98]. Interestingly, it has recently been shown that polyamine application on peach delays fruit ripening by interfering with ethylene production [99].

On the other hand, enzyme components generally involved in nucleoside phosphates turnover were also detected, namely acid phosphatase 1 (spot 42), soluble inorganic pyrophosphatase (spot 45), uridylate kinase (spot 69) and nucleoside diphosphate kinase 1(spot 97) species, the latter being also involved in signaling processes; they showed a progressive concentration decrease during apricot ripening. A similar quantitative trend for specific components has been already described in other fruit tissues $[12,32]$.

\subsection{Miscellaneous and unknown}

Different polypeptides that do not classify in the above categories were also identified as variably represented. For someone, a relation to defined physiological processes may be envisaged, but information on their quantitative profile during ripening of other fruits was practically absent. Among these, two protein species involved in RNA binding/processing were found to be down-represented upon ripening, namely RNA binding protein (spot 22) and regulator of ribonuclease activity a (spot 82). Other proteins involved in lipid transport showed a similar quantitative trend, i.e. acyl CoA binding protein (spot 100), tropism regulator membrane steroid binding protein (spots 56 and 57) [100] and temperature induced lipocalin (spot 76) species [101]. Although lipocalin function in plants is still poorly understood, they have been shown to increase in response to abiotic stress [102]. A progressive down-representation was also observed for other proteins whose functional role is still uncertain, being uncharacterized or assigned according to rough sequence homology criteria. They were uncharacterized protein components D7SK38, D9IZZ9, F6HZZ7 and C6SYI8 (spots 54, 55, 86 and 90, respectively), MFP1 attachment factor isoforms (spots 64 and 65), which are probably a structural component of plant nuclear matrix, elicitor responsive gene 3 (spot 71), which has been associated with pathogen defense and stress response in Triticum aestivum [103], and a thiosulfate sulfurtransferase species (spot 33), an enzyme containing rhodanese domains that catalyzes sulfur transfer to nucleophilic acceptors. Sulfurtransferase function in plants remains uncertain, but evidence has been provided that it may be involved in cellular redox homeostasis, probably acting as thioredoxin reductase [104]. In contrast, unnamed protein product F6I216 (spot 58) and stem-specific protein TSJT1 (spot 59) species showed a nine and four-fold increase at deep-orange stage, respectively.

\section{Concluding remarks}

This study provides a first attempt to shed light on the concentration changes that apricot proteins undergo during fruit 
development and ripening. This goal was accomplished by: i) the description of a large set of gene products expressed in fruit; ii) the achievement of a 2-D reference map; and iii) the identification of a group of ripening-related proteins. An obvious limitation to this investigation was the reduced genomic background available at this moment on this fruit; nevertheless, all the deregulated proteins were here positively characterized. The fact that, besides new candidates, many of the identified spots referred to proteins already known to be involved in the ripening of other fruits confirmed the reliability of our approach.

This investigation originally provides a preliminary overview of the important biological processes occurring during apricot maturation, which are in part schematically represented in Fig. 5. A functional classification of the protein species changing their concentration revealed the majority to be related to organic acids, carbohydrates and energy metabolism. The observed quantitative variations suggest a decrease in carbon fixation and photosynthesis-related steps at ripening, being the carbon skeleton demand to fuel the respiration burst accounted by an increase of glycolytic and fermentative pathways. Proteins involved in phytohormones metabolism and cell-wall restructuring, together with those associated with stress responses, including defense against pathogens, oxidative and abiotic ones were affected as well.
Their quantitative changes are fully consistent with an activation of the molecular machineries associated with climacteric fruit maturation and softening, nevertheless accounting also for some specific pomological parameter variations present during apricot ripening (Table 1). These preliminary results have to be further expanded by other gel-based or gel-free studies using alternative quantitative approaches (DIGE or ITRAQ TMT, ICAT, etc.), or integrated with complimentary proteomic investigations describing PTM changes (oxidation, phosphorylation, etc.) eventually associable to the physiological processes mentioned above. These proteomic studies, together with future investigations in the field of Rosaceae molecular biology, will certainly favor a deeper understanding of apricot fruit metabolism and its regulation during ripening, allowing for the development of new practices for fruit quality improvement.

Supplementary data to this article can be found online at http://dx.doi.org/10.1016/j.jprot.2012.11.008.

\section{Acknowledgments}

Authors thank Michele Carbone for his suggestions on apricot culture. This work was supported by a dedicated grant from the

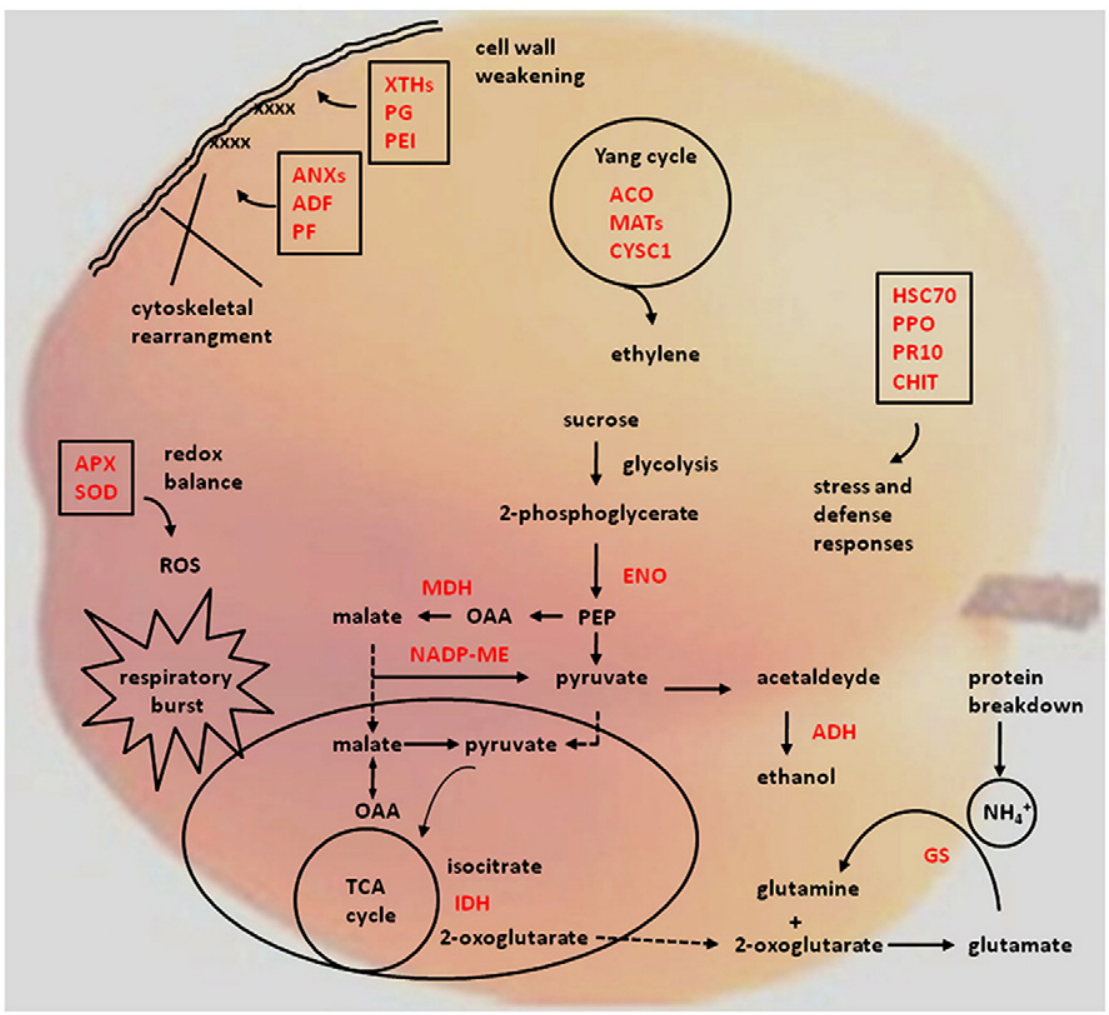

Fig. 5 - Schematic overview of the protein/enzyme species that changed their expression during the investigated stages of apricot ripening. Deregulated enzyme/protein species during ripening are indicated in red; related metabolites are also shown. ACO, 1-aminocyclopropane-1-carboxylate oxidase; ADH, alcohol dehydrogenase; ADF, actin depolymerising factor; ANX, annexin; APX, ascorbate peroxidase; CHIT, acidic endochitinase; CYSC1, $\beta$-cyanoalanine synthase; ENO, enolase; GS, glutamine synthetase; IDH, isocitric dehydrogenase; IFR, isoflavone reductase; MAT, S-adenosylmethionine synthetase; MDH, NAD-dependent malate dehydrogenase; NADP-ME, NADP-dependent malic enzyme; PEI, pectinesterase inhibitor; PG, endopolygalacturonase; PF, profilin; REM, remorin; SOD, superoxide dismutase; XTH, xyloglucan endotransglucosilase/ hydrolase. 
Italian Ministry of Economy and Finance to CNR for the project "Innovazione e sviluppo del Mezzogiorno - Conoscenze Integrate per Sostenibilità ed Innovazione del Made in Italy Agroalimentare" - Legge n.191/2009 and from funds "Progetto Agroalimentare, Ambiente e Salute - Intesa di Programma MIUR-CNR 2008-2011" to AS.

\section{R E F E R E N C E S}

[1] Vavilov NI. The origin, variation, immunity and breeding of cultivated plants. Chron Bot 1951;13:1-366.

[2] Zhebentyayeva T, Ledbetter CA, Burgos L, Llàcer G. Fruit Breeding. In: Badenes ML, Byrne PH, editors. Handbook of Plant Breeding, vol. 8. Springer; 2012. p. 415-58.

[3] Byrne DH, Littleton TG. Characterization of isoenzyme variability in apricots. J Am Soc Hortic Sci 1989;114: 674-8.

[4] Hagen LS, Khadari B, Lambert P, Audergon JM. Genetic diversity in apricot revealed by AFLP markers: species and cultivar comparisons. Theor Appl Genet 2002;105: 298-305.

[5] Zhebentyayeva T, Reighard L, Gorina M, Abbott G. Simple sequence repeat (SSR) analysis for assessment of genetic variability in apricot germplasm. Theor Appl Genet 2003;106: 433-44.

[6] Grimplet J, Romieu C, Audergon JM, Marty I, Albagnac G, Lambert P, et al. Transcriptomic study of apricot (Prunus armeniaca) ripening among 13006 expressed sequence tags. Physiol Plant 2005;125:281-92.

[7] Fernie AR, Stitt M. On the discordance of metabolomics with proteomics and transcriptomics: coping with increasing complexity in logic, chemistry, and network interactions scientific correspondence. Plant Physiol 2012;158:1139-45.

[8] Giovanoni JJ. Molecular biology of fruit maturation and ripening. Annu Rev Plant Physiol Plant Mol Biol 2001;57: 735-49.

[9] Carrari F, Fernie AR. Metabolic regulation underlying tomato fruit development. J Exp Bot 2006;57:1883-97.

[10] Palma JM, Corpas FJ, del Río LA. Proteomics as an approach to the understanding of the molecular physiology of fruit development and ripening. J Proteomics 2011;74: 1230-43.

[11] Faurobert M, Mihr C, Bertin N, Pawlowski T, Negroni L, Sommerer N, et al. Major proteome variations associated with cherry tomato pericarp development and ripening. Plant Physiol 2007;143:1327-46.

[12] Rocco M, D’Ambrosio C, Arena S, Faurobert M, Scaloni A, Marra M. Proteomic analysis of tomato fruits from two ecotypes during ripening. Proteomics 2006;6:3781-91.

[13] Giribaldi M, Perugini I, Sauvage FX, Schubert A. Analysis of protein changes during grape berry ripening by $2-\mathrm{DE}$ and MALDI-TOF. Proteomics 2007;7:3154-70.

[14] Deytieux C, Geny L, Lapaillerie D, Claverol S, Bonneu M, Donèche $\mathrm{B}$. Proteome analysis of grape skins during ripening. J Exp Bot 2007;58:1851-62.

[15] Negri AS, Prinsi B, Rossoni M, Failla O, Scienza A, Cocucci M, et al. Proteome changes in the skin of the grape cultivar Barbera among different stages of ripening. BMC Genomics 2008;9:378.

[16] Lücker J, Laszczak M, Smith D, Lund ST. Generation of a predicted protein database from EST data and application to ITRAQ analyses in grape (Vitis vinifera cv. Cabernet Sauvignon) berries at ripening initiation. BMC Genomics 2009;10:50.
[17] Martínez-Esteso MJ, Sellés-Marchart S, Lijavetzky D, Pedreño MA, Bru-Martínez R. A DIGE-based quantitative proteomic analysis of grape berry flesh development and ripening reveals key events in sugar and organic acid metabolism. J Exp Bot 2011;62:2521-69.

[18] Giribaldi M, Gény L, Delrot S, Schubert A. Proteomic analysis of the effects of ABA treatments on ripening Vitis vinifera berries. J Exp Bot 2010;61:2447-58.

[19] Sharathchandra RG, Stander C, Jacobson D, Ndimba B, Vivier MA. Proteomic analysis of grape berry cell cultures reveals that developmentally regulated ripening related processes can be studied using cultured cells. PLoS One 2011;6:e14708.

[20] Negri AS, Robotti E, Prinsi B, Espen L, Marengo E. Proteins involved in biotic and abiotic stress responses as the most significant biomarkers in the ripening of Pinot Noir skins. Funct Integr Genomics 2011;11:341-55.

[21] Muccilli V, Licciardello C, Fontanini D, Russo MP, Cunsolo V, Saletti R, et al. Proteome analysis of Citrus sinensis L. (Osbeck) flesh at ripening time. J Proteomics 2009;73:134-52.

[22] Pan Z, Zeng Y, An J, Ye J, Xu Q Deng X. An integrative analysis of transcriptome and proteome provides new insights into carotenoid biosynthesis and regulation in sweet orange fruits. J Proteomics 2012;75:2670-84.

[23] Lara MV, Borsani J, Budde CO, Lauxmann MA, Lombardo VA, Murray R, et al. Biochemical and proteomic analysis of 'Dixiland' peach fruit (Prunus persica) upon heat treatment. J Exp Bot 2009;60:4315-33.

[24] Qin G, Meng X, Wang Q Tian S. Oxidative damage of mitochondrial proteins contributes to fruit senescence: a redox proteomics analysis. J Proteome Res 2009;8:2449-62.

[25] Nilo R, Saffie C, Lilley K, Baeza-Yates R, Cambiazo V, Campos-Vargas $\mathrm{R}$, et al. Proteomic analysis of peach fruit mesocarp softening and chilling injury using difference gel electrophoresis (DIGE). BMC Genomics 2010;11:43.

[26] Zhang L, Yu Z, Jiang L, Jiang J, Luo H, Fu L. Effect of post-harvest heat treatment on proteome change of peach fruit during ripening. J Proteomics 2011;74:1135-49.

[27] Prinsi B, Negri AS, Fedeli C, Morgutti S, Negrini N, Cocucci M, et al. Peach fruit ripening: A proteomic comparative analysis of the mesocarp of two cultivars with different flesh firmness at two ripening stages. Phytochemistry 2011;72: 1251-62.

[28] Nilo RP, Campos-Vargas R, Orellana A. Assessment of Prunus persica fruit softening using a proteomics approach. J Proteomics 2012;75:1618-38.

[29] Bianco L, Lopez L, Scalone AG, Di Carli M, Desiderio A, Benvenuto E, et al. Strawberry proteome characterization and its regulation during fruit ripening and in different genotypes. J Proteomics 2009;72:586-607.

[30] Andrade JD, Toledo TT, Nogueira SB, Cordenunsi BR, Lajolo FM, do Nascimento JR. 2D-DIGE analysis of mango (Mangifera indica L.) fruit reveals major proteomic changes associated with ripening. J Proteomics 2012;75:3331-41.

[31] Nogueira SB, Labate CA, Gozzo FC, Pilau EJ, Lajolo FM. Oliveira do Nascimento JR. Proteomic analysis of papaya fruit ripening using 2DE-DIGE. J Proteomics 2012;75: 1428-1439.

[32] Huerta-Ocampo JÁ, Osuna-Castro JA, Lino-López GJ, Barrera-Pacheco A, Mendoza-Hernández G, De León-Rodríguez A, et al. Proteomic analysis of differentially accumulated proteins during ripening and in response to $1-\mathrm{MCP}$ in papaya fruit. J Proteomics 2012;75:2160-9.

[33] Costa F, Alba R, Schouten H, Soglio V, Gianfranceschi L, Serra $\mathrm{S}$, et al. Use of homologous and heterologous gene expression profiling tools to characterize transcription dynamics during apple fruit maturation and ripening. BMC Plant Biol 2010;10:229. 
[34] Qin G, Wang Q Liu J, Li B, Tian S. Proteomic analysis of changes in mitochondrial protein expression during fruit senescence. Proteomics 2009;9:4241-53.

[35] Leccese A, Bureau S, Reich M, Renard MG, Audergon JM, Mennone C, et al. Pomological and nutraceutical properties in apricot fruit: cultivation systems and cold storage fruit management. Plant Foods Hum Nutr 2010;65:112-20.

[36] Wang W, Vignani R, Scali M, Cresti M. A universal and rapid protocol for protein extraction from recalcitrant plant tissues for protomic analysis. Electrophoresis 2006;27: 2872-6.

[37] Ramagli LS, Rodriguez LV. Quantitation of microgram amounts of protein in two-dimensional polyacrylamide gel electrophoresis sample buffer. Electrophoresis 1985;6: 559-63.

[38] Talamo F, D'Ambrosio C, Arena S, Del Vecchio P, Ledda L, Zehender G, et al. Proteins from bovine tissues and biological fluids: defining a reference electrophoresis map for liver, kidney, muscle, plasma and red blood cells. Proteomics 2003;3:440-60.

[39] Scippa GS, Rocco M, Ialicicco M, Trupiano D, Viscosi V, Di Michele M, et al. The proteome of lentil (Lens culinaris Medik.) seeds: discriminating between landraces. Electrophoresis 2010;31:497-506.

[40] Peng J, Elias JE, Thoreen CC, Licklider LJ, Gygi SP. Evaluation of multidimensional chromatography coupled with tandem mass spectrometry (LC/LC-MS/MS) for large-scale protein analysis: the yeast proteome. J Proteome Res 2003;2: 43-50.

[41] Di Vaio C, Cirillo C, Lauro P, Ritieni A. Fruit quality evolution during ripening in apricot cultivars of Vesuvius area. Italus Hortus 2004;11:26-9.

[42] González-Agüero M, Troncoso S, Gudenschwager O, Campos-Vargas R, Moya-León MA, Defilippi BG. Differential expression levels of aroma-related genes during ripening of apricot (Prunus armeniaca L.). Plant Physiol Biochem 2009;47: 435-40.

[43] Berardinelli A, Cevoli C, Silaghi FA, Fabbri A, Ragni L, Giunchi A, et al. FT-NIR spectroscopy for the quality characterization of apricots (Prunus armeniaca L.). J Food Sci 2010;75:E462-8.

[44] Hegedüs A, Pfeiffer P, Papp N, Abrankó L, Blázovics A, Pedryc A, et al. Accumulation of antioxidants in apricot fruit through ripening: characterization of a genotype with enhanced functional properties. Biol Res 2011;44: 339-344.

[45] Brummel A, Dal Cin V, Crisosto CH, Labavitch JM. Cell wall meatbolism during maturation, ripening and senescence of peach fruit. J Exp Bot 2004;55:2029-39.

[46] Trainotti L, Zanin D, Casadoro G. A cell wall-oriented genomic approach reveals a new and unexpected complexity of the softening in peaches. J Exp Bot 2003;54:1821-32.

[47] Marty I, Bureau S, Sarkissian G, Gouble B, Audergon JM, Albagnac G. Ethylene regulation of carotenoid accumulation and carotenogenic gene expression in colour-contrasted apricot varieties (Prunus armeniaca). J Exp Bot 2005;56: 1877-86.

[48] Böttcher C, Keyzers RA, Boss PK, Davies C. Sequestration of auxin by the indole-3-acetic acid-amido synthetase GH3-1 in grape berry (Vitis vinifera L.) and the proposed role of auxin conjugation during ripening. J Exp Bot 2010;61: 3615-25.

[49] Symons GM, Chua YJ, Ross JJ, Quittenden LJ, Davies NW, Reid JB. Hormonal changes during non-climacteric ripening in strawberry. J Exp Bot 2012;63:4741-50.

[50] Else MA, Stankiewicz-Davies AP, Crisp CM, Atkinson CJ. The role of polar auxin transport through pedicels of Prunus avium L. in relation to fruit development and retention. J Exp Bot 2004;55:2099-109.
[51] Ludwig-Mueller J. Auxin conjugates: their role for plant development and in the evolution of land plants. J Exp Bot 2001;62:1757-73.

[52] Vizoso P, Meisel LA, Tittarelli A, Latorre M, Saba J, Caroca R, et al. Comparative EST transcript profiling of peach fruits under different post-harvest conditions reveals candidate genes associated with peach fruit quality. BMC Genomics 2009;10:423.

[53] Murayama H, Arikawa M, Sasaki Y, Dal Cin V, Mitsuhashi W, Toyomasu T. Effect of ethylene treatment on expression of polyuronide-modifying genes and solubilization of polyuronides during ripening in two peach cultivars having different softening characteristics. Postharvest Biol Technol 2009;52:196-201.

[54] Miedes E, Herbers K, Sonnewald U, Lorences EP. Overexpression of a cell wall enzyme reduces xyloglucan depolymerization and softening of transgenic tomato fruits. J Agric Food Chem 2010;58:5708-13.

[55] Mangeon A, Magioli C, Menezes-Salgueiro AD, Cardeal V, de Oliveira C, Costa-Galvao V, et al. AtGRP5 a vacuole-located glycine-rich protein involved in cell elongation. Planta 2009;230:253-65.

[56] Delgado IJ, Wang Z, de Rocker A, Keegstra K, Raikhel NV. Cloning and characterization of AtRGP1. A reversibly autoglycosylated protein implicated in cell wall biosynthesis. Plant Physiol 1998;118:1339-50.

[57] Staiger CJ, Yuan M, Valenta R, Shaw PJ, Warn R, Lloyd C. Microinjected profilin affects cytoplasmic streaming in plant cells by rapidly depolymerizing actin microfilaments. Curr Biol 1994;4:215-9.

[58] Thomas C, Tholl S, le Moes D, Dieterle M, Papuga J, Moreau F, et al. Actin bundling in plants. Cell Motil Cytoskeleton 2009;66:940-57.

[59] Blanchoin L, Boujemaa-Paterski R, Henty JL, Khurana P, Staiger CJ. Actin dynamics in plant cells: a team effort from multiple proteins orchestrates this very fast-paced game. Curr Opin Plant Biol 2010;13:714-23.

[60] Zhang JW, Ma HQ Feng JD, Zeng L, Wang Z, Chen SW. Grape berry plasma membrane proteome analysis and its differential expression during ripening. J Exp Bot 2008;59: 2979-90.

[61] Vantard M, Blanchoin L. Actin polymerization processes in plant cells. Curr Opin Plant Biol 2002;5:502-6.

[62] Le LQ, Mahler V, Scheurer S, Foetisch K, Braun Y, Weigand $\mathrm{D}$, et al. Yeast profiling complement profilin deficiency in transgenic tomato fruits and allows development of hypoallergenic tomato fruits. FASEB J 2010;24:4939-47.

[63] Lee JM, Kusakabe T, Kawaguchi Y, Miyagawa Y, Takahashi M, Mon H, et al. Molecular cloning and characterization of the translationally controlled tumor protein gene in Bombyx mori. Comp Biochem Physiol B 2004;139:35-43.

[64] Barba-Espin G, Diaz-Vivancos P, Clemente-Moreno MJ, Albacete A, Faize L, Faize M, et al. Interaction between hydrogen peroxide and plant hormones during germination and the early growth of pea seedlings. Plant Cell Environ 2010;33:981-94.

[65] Gerke V, Creutz CE, Moss SE. Annexins: Linking $\mathrm{Ca}^{2+}$ signalling to membrane dynamics. Nat Rev Mol Cell Biol 2005;6:449-61.

[66] Lu Y, Ouyang B, Zhang J, Wang T, Lu C, Han Q et al. Genomic organization, phylogenic comparison and expression profiles of annexin gene family in tomato (Solanum lycopersicum). Gene 2012;4991:14-24.

[67] Mortimer JC, Laohavisit A, Macpherson N, Webb A, Brownlee C, Battey NH, et al. Annexins: multifunctional components of growth and adaptation. J Exp Bot 2008;59: 533-44. 
[68] Jimenez A, Creissen G, Kular B, Firmin J, Robinson S, Verhoeyen $\mathrm{M}$, et al. Changes in oxidative processes and components of the antioxidant system during tomato fruit ripening. Planta 2002;214:751-8.

[69] Camejo D, Martì MC, Roman P, Ortiz A, Jimenez A. Antioxidant system and protein pattern in peach fruits at two maturation stages. J Agric Food Chem 2010;58: 11140-11147.

[70] Navrot N, Collin V, Gualberto J, Gelhaye E, Hirasawa M, Rey $\mathrm{P}$, et al. Plant glutathione peroxidases are functional peroxiredoxines distributed in several subcellular compartments and regulated during biotic and abiotic stress. Plant Physiol 2006;142:1364-79.

[71] Paganga G, Miller N, Rice-Evans CA. The polyphenolic content of fruit and vegetables and their antioxidant activities. What does a serving constitute? Free Radic Res 1999;30:153-62.

[72] Brkljacic J, Zhao Q Meier I. WWP-domain proteins mimic the activity of the HSC70-1chaperone in preventing mistargeting of Ran-Gap1-anchoring protein WIT1. Plant Physiol 2009;151:142-54.

[73] Huang C, Verrillo F, Renzone G, Arena A, Rocco M, Scaloni A, et al. Response to biotic and oxidative stress in Arabidopsis thaliana: analysis of variably phosphorylated proteins. J Proteomics 2011;74:1934-49.

[74] Henry MI, Carpentier SC, Pampurova S, van Hoylandt A, Panis B, Swennen R, et al. Structure and regulation of Asr gene family in banana. Planta 2011;234:785-98.

[75] Kim IS, Kim YS, Yoon HS. Rice ASR1 protein with reactive oxygen species scavenging and chaperone-like activities enhances acquired tolerance to abiotic stresses in Saccharomyces cerevisiae. Mol Cells 2012;33: 285-93.

[76] Fulda S, Mikkat S, Stegmann H, Horn R. Physiology and proteomics of drought stress acclimation in sunflower (heliantus annuus L.). Plant Biol 2011;13:632-42.

[77] Kim JY, Park SJ, Jang B, Jung CH, Ahn SJ, Goh CH, et al. Functional characterization of a glycine-rich RNA-binding protein 2 in Arabidopsis thaliana under abiotic stress conditions. Plant J 2007;50:439-51.

[78] Patil DN, Datta M, Chaudhary A, Tomar S, Sharma AK, Kumar P. Isolation, purification, crystallization and preliminary crystallographic studies of chitinase from tamarind (Tamarindus indica) seeds. Acta Crystallogr 2009; F65:343-5.

[79] Guarino C, Arena S, De Simone L, D’Ambrosio C, Santoro S, Rocco M, et al. Proteomic analysis of the major soluble components in Annurca apple flesh. Mol Nutr Food Res 2007;51:255-62.

[80] Brenna OV, Pastorello EA, Farioli L, Pravettoni V, Pompei C. Presence of allergenic proteins in different peach (Prunus persica) cultivars and dependance of their content on fruit ripening. J Agric Food Chem 2004;52:7997-8000.

[81] Jarsch IK, Ott T. Perspectives on remorin proteins, membrane rafts, and their role during plant-microbe interactions. Mol Plant Microbe Interact 2011;24:7-12.

[82] Mayer AM, Harel E. Phenoloxidases and their significance in fruit and vegetables. In: Fox PF, editor. Food Enzymology. Elsevier; 1991. p. 373-98.

[83] Shoji T, Winz R, Iwase T, Keiji Nakajima K, Yamada Y, et al. Expression patterns of two tobacco isoflavone reductase-like genes and their possible roles in secondary metabolism in tobacco. Plant Mol Biol 2002;50:427-40.

[84] Babiychuk E, Kushnir S, Belles-Boix E, Van Montagu M, Inze D. Arabidposis thaliana NADPH oxidoreductase homologs confer tolerance of yeast toward the thiol-oxidizing drug diamide. J Biol Chem 1995;270:26224-31.

[85] Kim SG, Kim ST, Wang Y, Kim SK, Lee CH, Kim KK, et al. Overexpression of rice isoflavone reductase-like gene
(OsIRL) confers tolerance to reactive oxygen species. Physiol Plant 2010;138:1-9.

[86] Raab T, Lopez-Ra JA, Klein D, Caballero JL, Moyano E, Schwab $\mathrm{W}$, et al. FaQR, required for the biosynthesis of the strawberry flavor compound 4-hydroxy-2,5-dimethyl 3(2H) furanone, encodes an enone oxidoreductase. Plant Cell 2006;18:1023-7.

[87] Etienne C, Moing A, Dirlewanger E, Raymond P, Monet R, Rotan C. Isolation and characterization of six peach cDNAs key proteins in organic acid metabolism and solute accumulation: involvement in regulating peach fruit acidity. Physiol Plant 2002;114:259-70.

[88] Sweetman C, Deluc LG, Cramer GR, Ford CM, Soole KL. Regulation of malate metabolism in grape berry and other developing fruits. Phytochemistry 2009;70:1329-44.

[89] Vand der Straeten D, Rodrigues-Pousada RA, Goodman HM, Van Montagua M. Plant enolase: gene structure, expression and evolution. Plant Cell 1991;3:719-35.

[90] Borsani J, Budde CO, Porrini L, Lauxmann MA, Lombardo V, Murray R, et al. Carbon metabolism of peach fruit harvest: changes in enzymes involved in organic acid and sugar levels modifications. J Exp Bot 2009;60: 1823-37.

[91] Kan J, Wang HM, Jin CH, Xie HY. Changes of reactive oxygen species and related enzymes in mitochondria respiratory metabolism during the ripening of peach fruit. Agric Sci China 2010;9:138-46.

[92] Giovannoni JJ. Genetic regulation of fruit development and ripening. Plant Cell 2004;16:S170-80.

[93] Egea I, Barsan C, Bian W, Purgatto E, Latche A, Chervin C, et al. Chromoplast differentiation: current status and perspectives. Plant Cell Physiol 2010;51:1601-11.

[94] Marti MC, Camejo D, Olmos E, Sandalio LM, Fernandez-Garcia N, Jimenez A, et al. Characterisation and changes in the antioxidant system of chloroplasts and chromoplasts isolated from green and mature pepper fruits. Plant Biol 2009;11:613-24.

[95] Chang IF, Chen PJ, Shen CH, Hsieh TJ, Hsu YW, Huang BL, et al. Proteomic profiling of proteins associated with the rejuvenation of Sequoia sempervirens (D. Don) Endl. Proteome Sci 2010;8:64.

[96] Lombardo VA, Osorio S, Borsani J, Lauxmann MA, Bustamante CA, Budde CO, et al. Metabolic profiling during peach fruit development and ripening reveals the metabolic networks that underpin each developmental stage. Plant Physiol 2011;157:1696-710.

[97] Miflin BJ, Habash DZ. The role of glutamine synthetase and glutamate dehydrogenase in nitrogen assimilation and possibilities for improvement in the nitrogen assimilation of crops. J Exp Bot 2002;53:978-87.

[98] Wimalasekera R, Tebartz F, Scherer GF. Polyamines, polyamine oxidases and nitric oxide in development, abiotic and biotic stresses. Plant Sci 2011;181:593-603.

[99] Torrigiani P, Bressanin D, Beatriz Ruiz K, Tadiello A, Trainotti L, Bonghi C, et al. Spermidine application to young developing peach fruits leads to a slowing down of ripening by impairing ripening-related ethylene and auxin metabolism and signaling. Physiol Plant 2012;146: 86-98.

[100] Yang X, Song L, Xue HW. Membrane steroid binding protein 1 (MSBP1) stimulates tropism by regulating vesicle trafficking and auxin redistribution. Mol Plant 2008;1: 1077-87.

[101] Flower DR. The lipocalin protein family: structure and function. Biochem J 1996;318:1-14.

[102] Charron JF, Breton G, Badawi M, Sarhan F. Molecular and structural analyses of a novel temperature stress-induced lipocalin from wheat and arabidopsis F. FEBS Lett 2002;517: 129-32. 
[103] Zhang G, Xia N, Kang Z. TaERG3: a novel elicitor responsive gene implicated in resistance to stripe rust pathogen infection and abiotic stresses. EMBL/GenBank/DDBJ databases. submitted for publication.

[104] Guretzki S, Papenbrock J. Characterization of the sulfurtransferase family from Oryza sativa L. Plant Physiol Biochem 2011;49:1064-70.
[105] Bevan M, Bancroft I, Bent E, Love K, Goodman H, Dean C, et al. Analysis of $1.9 \mathrm{Mb}$ of contiguous sequence from chromosome 4 of Arabidopsis thaliana. Nature 1998;391: 485-8. 